Notre Dame Journal of Formal Logic

Volume 26, Number 4, October 1985

\title{
Two $\lambda$-Extensions of the Theory of Homogeneous Simple Types as a Second-Order Logic
}

\author{
NINO B. COCCHIARELLA
}

In the theory of simple logical types as originally conceived, it is meaningless for one predicate expression to occur in one of the subject or argument positions of another unless the latter is assigned a higher logical type than the former within the grammar of the object language; and therefore it is meaningless in particular for any predicate expression to apply to itself, i.e., to occur in one of its own subject positions. Russell's paradox of predication is thereby avoided, of course, but the price is high, for the resulting theory is not an accurate representation of the role of predicates in natural language where predicate expressions can apply not only to the nominalizations of other predicates but to their own nominalizations as well-and without regard at all for the notion of a logical type.

In the theory of logical types as a second-order logic, on the other hand, predicate expressions are typed within the grammar of the object language only in the way they are typed in standard second-order logic, i.e., only with respect to their degree or number of subject positions (adicity), and they are allowed otherwise to meaningfully occur in the subject or argument positions of other predicates, and of themselves as well, without regard to the notion of a logical type. Russell's paradox of predication can be avoided, it turns out, not by resorting to the notion of a logical type as a part of the grammar of the object language but rather only as a part of the metalinguistic description of the conditions under which properties and relations are to be posited by means of the grammar of the object language. The difference is crucial, needless to say, since it allows for a more accurate representation of the role of predicates and predication in natural language. The resulting theory is not, to be sure, a secondorder logic in the "standard" sense used today (though it does contain the latter), but it is a second-order logic in the traditional or pre-type-theoretical sense in which quantifier expressions are allowed to reach into both subject and 
predicate positions without obliterating the logical and conceptually important distinctions between the two.

We return in the present essay to the theory of homogeneous simple types (HST) as a second-order logic, and in particular to the theory $H S T^{*}$ described in [2]. We shall be concerned only with homogeneous simple types since, as was noted in [2], the full relational theory $S T^{*}$ of heterogeneous simple types is inconsistent as a second-order logic. We shall extend the grammar of $H S T^{*}$, however, by introducing the $\lambda$-operator as a new logical primitive, thereby allowing for the formation of $\lambda$-abstracts as complex predicate expressions. Our first goal is to formulate a system $\lambda H S T^{*}$ which together with a principle of individuation for properties and relations is a conservative extension of $H S T^{*}$ (with that same principle of individuation); and in fact the system we formulate is not only a conservative extension but an inessential one since $\lambda$-abstraction is in principle eliminable therein. Unfortunately, however, the realization of this goal requires that we constrain the grammar of the object language and allow as meaningful only those $\lambda$-abstracts which are homogeneously stratified (in the metalinguistic sense); i.e., we must impose on the grammar of the object language for $\lambda H S T^{*}$ just those conditions which in $H S T^{*}$ applied only to the conditions for positing properties and relations. A secondary goal, accordingly, is to formulate a system $H S T_{\lambda}^{*}$ which does not impose these grammatical constraints on $\lambda$-abstracts but which posits as individuals only those properties and relations posited in $H S T^{*}$ - and of course which is consistent if $H S T^{*}$ is, even though it is not a conservative extension of $H S T^{*}$. This is achieved in part by rendering the first-order logic contained in $H S T_{\lambda}^{*}$ free of existential presuppositions and allowing some $\lambda$-abstracts when occurring in subject positions to denote a value of the bound individual variables while admitting that others, of necessity, will fail to do so.

As a means towards realizing these goals, we reformulate the extensional Fregean semantics given for $H S T^{*}$ in [2], and we show that both $\lambda H S T^{*}$ and $H S T_{\lambda}^{*}$, together with an extensional principle of individuation for properties and relations, are complete with respect to their extensional Fregean semantics. (A Fregean semantics, as we understand it here, is one in which nominalized predicates are interpreted as denoting certain individuals called concept-correlates rather than the concepts designated by these predicates in their predicate position occurrences.) We also intensionalize this semantics in a concluding section and show that two modal extensions of $\lambda H S T^{*}$ and $H S T_{\lambda}^{*}$, together with an intensional principle of individuation for properties and relations, are complete with respect to this intensionalized Fregean semantics.

Finally, it should be noted that as a counterpart to the original theory of simple logical types much of the motivation for our development of $\lambda H S T^{*}$ and $H S T_{\lambda}^{*}$ parallels Quine's motivation for the construction of his two first-order set theories, viz., the system $N F$ of [5] and the later system $M L$ of [6]. Moreover, in this regard, even our proof of the consistency of $H S T_{\lambda}^{*}$ relative to $\lambda H S T^{*}$ parallels Wang's proof in [7] of the consistency of $M L$ relative to $N F$. Nevertheless, notwithstanding this similarity and parallel motivation, the differences in deductive powers between $N F$ and $M L$, on the one hand, and $\lambda H S T^{*}$ and $H S T_{\lambda}^{*}$, on the other, are not insignificant. For example, as shown in [2], $H S T^{*}$, and therefore $\lambda H S T^{*}$ as well, is equiconsistent with the original theory of simple 
logical types, $S T$, at least when both systems are supplemented with an extensional principle of individuation for properties and relations. $N F$, on the other hand, not only exceeds $S T$ in deductive powers but does so in inappropriate ways, e.g., by disproving the axiom of choice and thereby proving an axiom of infinity. Neither of these axioms, however, is either provable or disprovable in $S T, \lambda H S T^{*}$, or $H S T_{\lambda}^{*}$. In fact, unlike $N F$ and $M L$, both $\lambda H S T^{*}$ and $H S T_{\lambda}^{*}$ (with and without axioms of choice and infinity) as well as $S T$ can be shown to be consistent if weak Zermelo set theory (with and without axioms of choice and infinity) is consistent. In other words, as theories of predication, $\lambda H S T^{*}$ and $H S T_{\lambda}^{*}$ are better type-free representations of the original theory of simple logical types than is either $N F$ or $M L$ as a theory of membership. Moreover, many, if not all, of the controversial theses of $N F$ and $M L$ as set theories independent of any superseding theory of predication (e.g., their admission of a universal set and of the set-existence of the complement of every set, and their countenance therefore of a symmetry between the small and the large) are neither dubious nor controversial in $\lambda H S T^{*}$ and $H S T_{\lambda}^{*}$ when viewed as theses about properties and relations in a theory of predication. Finally, even the problematic distinction between proper classes and sets in $M L$ has a natural explanation when rephrased in terms of the distinction in $H S T_{\lambda}^{*}$ between those $\lambda$-abstracts which denote a value of the bound individual variables and those which do not (even though all $\lambda$-abstracts designate or express values of bound predicate variables). In particular, whereas nominalized predicates may be taken as always purporting to denote an independently real property or relation (though some of necessity must fail to denote anything at all), the use of these same predicates in their role as predicates, i.e., their occurrences in predicate positions, may be assumed always to express only unsaturated predicable concepts in the sense of (mind dependent) cognitive capacities (or cognitive structures otherwise based upon such capacities). That is, $H S T_{\lambda}^{*}$ can be construed as representing a form of conceptual realism where the use of a predicate as such amounts to the application of an (unsaturated) predicable concept in an act of thought (wherein the concept becomes saturated), but also where the denotative use of the same predicate, i.e., the use of its nominalization, amounts instead to an attempt to refer to a real property or relation which is purportedly represented by the concept expressed by that predicate. That not every predicable concept which we can form and articulate can succeed in denoting a real property or relation when applied denotatively will in that case be an interesting but hardly problematic fact. What would be problematic is the assumption that concepts are themselves the independently real properties and relations they purport to represent when applied denotatively while admitting that only some of them are individuals while others are not. Given that assumption, in other words, it would be better to stick with $\lambda H S T^{*}$ where (at least on its intended interpretation) all values of bound predicate variables are also values of the bound individual variables.

$1 \mathrm{HST}^{*}$ revisited We begin by reviewing the syntax of $H S T^{*}$. This consists essentially of the wffs of standard second-order logic extended so as to allow predicate variables and constants to occupy the subject or argument positions that go with predicates. Predicates occupying such positions are said to be 
nominalized (at such positions) and are understood to represent the predicate nominalizations that occur in natural language. (Adding suffixes such as '-ity', '-ness', or '-hood' to nominalized occurrences of predicates would be superfluous here, incidentally, since such occurrences are already formally identified as subject position occurrences.)

We shall use ' $x$ ', ' $y$ ', ' $z$ ', with or without numerical subscripts, to refer (in the metalanguage) to individual variables, and we use ' $F^{n}$ ' and ' $G{ }^{n}$ ' similarly to refer to $n$-place predicate variables. (We shall usually delete the superscript when the context makes clear the number of subject or argument positions that go with a predicate variable or constant.) We shall also use ' $a$ ' and ' $b$ ', with or without numerical subscripts, to refer ambiguously (in the metalanguage) to both predicate and individual variables and constants. Predicate and individual constants we assume to be provided by different (formal) languages; i.e., a language is a set of predicate and individual constants.

As primitive logical constants, we take $\rightarrow$, the (material) conditional sign, $\sim$, the negation sign, and $\forall$, the universal quantifier. (The other logical constants, viz., \&, $\vee, \leftrightarrow$, and $\exists$, are assumed to be defined as abbreviatory devices of the metalanguage.) Where $L$ is a language, we understand the terms of $L$ to be the members of $L$ together with all individual and predicate variables. The atomic wffs of $L$ are all the expressions of the form: $P^{n}\left(a_{1}, \ldots, a_{n}\right)$, where $n \in \omega, P^{n}$ is either an $n$-place predicate constant in $L$ or an $n$-place predicate variable, and $a_{1}, \ldots, a_{n}$ are terms of $L$. (When $n=0$, we take $P^{n}\left(a_{1}, \ldots, a_{n}\right)$ to be just $P^{n}$ itself.) An expression is a $w f f$ of $L$ iff it belongs to every set $K$ containing the atomic wffs of $L$ and such that $\sim \phi,(\phi \rightarrow \psi), \forall a \phi$ are in $K$ whenever $\phi, \psi$ are in $K$ and $a$ is an individual or predicate variable. We use ' $\phi$ ', ' $\psi$ ', and ' $\chi$ ' to refer (in the metalanguage) to wffs.

We assume the notions of bondage and freedom of occurrences of variables in wffs to be defined in the usual way, and similarly we assume what it means for a term $a$ to be free for a term $b$ in a wff $\phi$. We take $\phi(a / b)$ to be the result of replacing all free occurrences of $b$ in $\phi$ by free occurrences of $a$, if such a wff exists; and otherwise we take $\phi(a / b)$ to be just $\phi$ itself. For convenience, we use $\equiv$ to abbreviate (in the metalanguage) indiscernibility as follows (where $F$ is the first one-place predicate variable different from both $a$ and $b$ ):

$$
a \equiv b=_{d f} \forall F[F(a) \leftrightarrow F(b)] .
$$

Finally, we say that a wff $\phi$ is homogeneously stratified if there is an assignment $t$ of natural numbers to the terms that occur in $\phi$ such that for every subwff of $\phi$ of the form $P^{n}\left(a_{1}, \ldots, a_{n}\right)$ : (1) $t\left(a_{i}\right)=t\left(a_{j}\right)$, for all positive integers $i, j \leq n$, and (2) $t\left(P^{n}\right)=1+t\left(a_{1}\right)$.

2 An improved axiom set for $\boldsymbol{H S T}$ * The axiomatization of $H S T^{*}$ given in [2] can be improved, it turns out, in several ways that are conducive to the formulation of the systems $\lambda H S T^{*}$ and $H S T_{\lambda}^{*}$. Briefly, the original axioms of $H S T^{*}$ consisted of all tautologous wffs and all wffs of one of the following forms:

(A1) $\forall a(\phi \rightarrow \psi) \rightarrow(\forall a \phi \rightarrow \forall a \psi)$, where $a$ is any variable

(A2) $\phi \rightarrow \forall a \phi$, where $a$ is a variable not occurring free in $\phi$ 
(A3) $\exists x(a \equiv x)$, where $a$ is any term in which the individual variable $x$ does not occur free

(A4) $\exists F^{n}\left(P \equiv F \& \forall x \ldots \forall x_{n}\left[F\left(x_{1}, \ldots, x_{n}\right) \leftrightarrow P\left(x_{1}, \ldots, x_{n}\right)\right]\right)$, where $x_{1}, \ldots, x_{n}$ are pairwise distinct individual variables, $P^{n}$ is an $n$-place predicate variable or constant, and $F^{n}$ is an $n$-place predicate variable different from $P^{n}$

(A5) $a \equiv b \rightarrow(\phi \rightarrow \psi)$, where $\phi$ is an atomic wff and $\psi$ is obtained from $\phi$ by replacing a subject position occurrence of $b$ by a subject position occurrence of $a$,

together with all homogeneously stratified wffs that are instances of the following comprehension principle:

$\left(\mathbf{H S C P}^{*}\right) \quad \exists F^{n} \forall a_{1} \ldots \forall a_{n}\left[F\left(a_{1}, \ldots, a_{n}\right) \leftrightarrow \phi\right]$,

where $F^{n}$ is an $n$-place predicate variable not occurring free in $\phi$ and $a_{1}, \ldots, a_{n}$ are pairwise distinct individual or predicate variables occurring free in $\phi$. (Note: the entire biconditional must be homogeneously stratified in this principle, not just the comprehending wff $\phi$ alone. Also, in general, we use a *-label for any thesis which explicitly involves predicate nominalizations.)

The only inference rules of $H S T^{*}$ (and later of $\lambda H S T^{*}$ and $H S T_{\lambda}^{*}$ as well) are modus ponens (MP) and universal generalization (UG) of either a predicate or individual variable. Theoremhood is assumed defined in the usual way, and $\Gamma \vdash_{H S T^{*}} \phi$ is taken to mean that $\vdash_{H S T^{*}}\left(\psi_{1} \& \ldots \& \psi_{n} \rightarrow \phi\right)$, for some $\psi_{1}, \ldots$, $\psi_{n} \in \Gamma$. (For $n=0$, we take the above "conditional" to be just $\phi$ itself.) As observed in [2], the following theses are provable without utilizing $\left(\mathrm{HSCP}^{*}\right)$ at all:

(1) Leibniz' law for subject position occurrences of terms:

$\left(\mathbf{L L}_{1}^{*}\right) \quad a \equiv b \rightarrow(\phi \leftrightarrow \psi)$,

where $\psi$ is obtained from $\phi$ by replacing one or more free subject position occurrences of a by free subject position occurrences of $b$.

(2) The principle of universal instantiation of any term a for a generalized individual variable $x$ :

(UI) $\quad \forall x \phi \rightarrow \phi(a / x)$.

(3) The principle of universal instantiation of an n-place predicate variable or constant $P^{n}$ for a generalized $n$-place predicate variable:

(UI $\mathbf{I}_{\text {n }}^{*} \quad \forall F^{n} \phi \rightarrow \phi\left(P^{n} / F\right)$.

Now because

$\exists R \forall F \forall G\left(R(F, G) \leftrightarrow \forall x_{1} \ldots \forall x_{n}\left[F\left(x_{1}, \ldots, x_{n}\right) \leftrightarrow G\left(x_{1}, \ldots, x_{n}\right)\right]\right)$

is an instance of $\left(H S C P^{*}\right)$, the following thesis,

(Ind In $^{*} \quad \forall F^{n} \forall G^{n}\left(F \equiv G \rightarrow \forall x_{1} \ldots \forall x_{n}\left[F\left(x_{1}, \ldots, x_{n}\right) \leftrightarrow G\left(x_{1}, \ldots, x_{n}\right)\right]\right)$ 
is provable in $H S T^{*}$; and therefore, by a simple induction on the subwffs of $\phi$, the above restricted version of Leibniz' law can be strengthened into the full version:

(LL $\left.\mathbf{L}^{*}\right) \quad \vdash_{H S T^{*}} a \equiv b \rightarrow(\phi \leftrightarrow \psi)$,

where $\psi$ is obtained from $\phi$ by replacing one or more free occurrences of $a$ by free occurrences of $b$ (if such a wff exists, and otherwise $\psi$ is just $\phi$ itself $^{1}$ ). Indiscernibility in $H S T^{*}$, in other words, suffices as a characterization of identity.

$\left(L L^{*}\right)$ is also derivable of course if we simply drop the restriction in (A5) that only subject position occurrences of a term are to be replaced under the hypothesis of indiscernibility. (Note: although unimportant in $H S T^{*}$, the restriction is crucial in certain other theories, specifically the system $T^{*}\left({ }^{*}\right)$ of Section 2 in [2], where $\left(I n d_{n}^{*}\right)$ is disprovable; i.e., the distinction is crucial in theories in which indiscernibility necessarily fails as a characterization of identity.) Strengthening (A5) in this way, needless to say, allows us to derive $\left(I n d^{*}\right)$ without resorting to $\left(H S C P^{*}\right)$ at all. (We take $\left(I n d^{*}\right)$ to be the set of wffs $\left(I n d_{n}^{*}\right)$, for all $\left.n \in \omega\right)$. Now this is significant because given $\left(I n d^{*}\right)$, we can weaken or rather restrict $\left(H S C P^{*}\right)$ to just those homogeneously stratified wffs of the form:

$$
\exists F^{n} \forall x_{1} \ldots \forall x_{n}\left[F\left(x_{1}, \ldots, x_{n}\right) \leftrightarrow \phi\right],
$$

where $F_{n}$ is not free in $\phi$ and $x_{1}, \ldots, x_{n}$ are pairwise distinct individual variables occurring free in $\phi$. That is, the original quantifier prefix $\forall a, \ldots, \forall a_{n}$ of $\left(H S C P^{*}\right)$ can be restricted to those cases where none of $a_{1}, \ldots, a_{n}$ is a predicate variable.

Proof: Suppose

$$
F\left(a_{1}, \ldots, a_{n}\right) \leftrightarrow \phi
$$

is a homogeneously stratified wff as in the original version of $\left(H S C P^{*}\right)$. Let $x_{1}, \ldots, x_{n}$ be distinct individual variables new to $\phi$, and let $b_{i}$ be a variable new to $\phi$ but of the same type as $a_{i}$. Then, on the basis of $\left(L L^{*}\right)$ and the remaining axioms of $H S T^{*}$ other than $\left(H S C P^{*}\right)$,

$$
\phi \leftrightarrow \exists b, \ldots, \exists b_{n}\left[a_{1} \equiv b_{1} \& \ldots \& a_{n} \equiv b_{n} \& \phi\left(b_{1} / a_{1}, \ldots, b_{n} / a_{n}\right)\right]
$$

is provable; and since

$$
\begin{gathered}
\forall x_{1} \ldots \forall x_{n}\left[F ( x _ { 1 } , \ldots , x _ { n } ) \leftrightarrow \exists b _ { 1 } \ldots \exists b _ { n } \left[x_{1} \equiv b_{1} \& \ldots \& x_{n} \equiv b_{n} \&\right.\right. \\
\left.\left.\phi\left(b_{1} / a_{1}, \ldots, b_{n} / a_{n}\right)\right]\right) \rightarrow \\
\left(F\left(a_{1}, \ldots, a_{n}\right) \leftrightarrow \exists b_{1} \ldots \exists b_{n}\left[a_{1} \equiv b_{1} \& \ldots \& a_{n} \equiv b_{n} \& \phi\left(b_{1} / a_{1}, \ldots, b_{n} / a_{n}\right)\right]\right)
\end{gathered}
$$

is provable on the basis of $\left(U I_{1}^{*}\right)$ (which does not itself depend on $\left(H S C P^{*}\right)$ ), then by the interchange law for wffs provably equivalent (which also does not depend on $\left.\left(H S C P^{*}\right)\right)$, universal generalization on $a_{1}, \ldots, a_{n}, F_{n}$, and tautologous transformations utilizing $(A 1)$ and $(A 2)$,

$$
\begin{gathered}
\exists F \forall x_{1} \ldots \forall x_{n}\left(F ( x _ { 1 } , \ldots , x _ { n } ) \leftrightarrow \exists b _ { 1 } \ldots \exists b _ { n } \left[x_{1} \equiv b_{1} \& \ldots \& x_{n} \equiv b_{n} \&\right.\right. \\
\left.\left.\phi\left(b_{1} / a_{1}, \ldots, b_{n} / a_{n}\right)\right]\right) \rightarrow \\
\exists F \forall a_{1} \ldots \forall a_{n}\left[F\left(a_{1}, \ldots, a_{n}\right) \leftrightarrow \phi\right]
\end{gathered}
$$


is also provable without utilizing $\left(H S C P^{*}\right)$. Of course since by hypothesis the consequent of this last conditional is an instance of the original version of $\left(H S C P^{*}\right)$, it follows that the antecedent is also an instance of $\left(H S C P^{*}\right)$, and therefore of the restricted version in question as well. That is, on the basis of $\left(L L^{*}\right)$ and the remaining axioms of $H S T^{*}$, the original version of $\left(H S C P^{*}\right)$ is derivable from the restricted version.

Hereafter, in referring to $H S T^{*}$ we shall take this to be the system described above with $\left(H S C P^{*}\right)$ replaced by its restricted version and $(A 5)$ replaced by $\left(L L^{*}\right)$ (or by $(A 5)$ strengthened in the way indicated). This is an improvement over the original version, incidentally, if only because it explains why definitionally extending an applied form of $H S T^{*}$ by means of an explicit definition for a predicate constant must have the form of a homogeneously stratified biconditional:

$$
\forall x_{1} \ldots \forall x_{n}\left[P^{n}\left(x_{1}, \ldots, x_{n}\right) \leftrightarrow \phi\right],
$$

where $\phi$ is a wff in which the $n$-place predicate constant $P^{n}$ does not occur, $x_{1}, \ldots, x_{n}$ are distinct individual variables occurring free in $\phi$, and no variables other than $x_{1}, \ldots, x_{n}$ occur free in $\phi$. That is, as is appropriate for any secondorder logic with nominalized predicates, the defining conditions for a new predicate must always be such as to be meaningful for all individuals, whether these be concrete or abstract, and not just for individuals of a certain type (as might have been thought on the basis of the original form of $\left.\left(H S C P^{*}\right)\right)$. Definitions of this form, needless to say, can easily be shown to be noncreative, and defined predicates are provably eliminable on the basis of the restricted version of $\left(H S C P^{*}\right)$.

3 The grammar of $\boldsymbol{H S T}^{*}$ with $\lambda$-abstracts Given the $\lambda$-operator and the fact that we are dealing with a logic with nominalized predicates, explicit definitions of predicate constants can be given the even more natural form of an identity sentence:

$$
P^{n} \equiv\left[\lambda x_{1}, \ldots, x_{n} \phi\right],
$$

where $\left[\lambda x_{1} \ldots x_{n} \phi\right]$ is a homogeneously stratified $\lambda$-abstract. Note that only individual variables are allowed to be bound by the $\lambda$-operator, since to do otherwise might suggest that we have predicate expressions which on grammatical grounds alone cannot be meaningfully predicated of all individuals, thereby defeating the whole point of reconstructing type theory as a second-order logic. Individual variables, in other words, are subject position indicators par excellence.

Now in taking the $\lambda$-operator as a new primitive logical constant, we shall describe the syntax of $\lambda H S T^{*}$ in terms of the somewhat broader syntax of $H S T_{\lambda}^{*}$, i.e., in terms of a syntax which does not require all $\lambda$-abstracts to be homogeneously stratified. For convenience, we identify the (category) types of the syntax with the set of natural numbers, where semantically 0 is understood to be the type of all individuals, 1 is the type of all propositions, 2 is the type of all properties, and $n+1$, for $n>1$, the type of all $n$-ary relations. Accordingly, individual variables are said to be of type 0 , and $n$-place predicate 
variables are said to be of type $n+1$. Where $L$ is a language and $n \in \omega$, then the meaningful expressions of $L$ of type $n$, in symbols $M E_{n}(L)$, are recursively defined as follows:

(1) if $a$ is an individual variable or constant in $L$, then $a \in M E_{0}(L)$; and if $a$ is an $n$-place predicate variable or constant in $L$, then $a \in M E_{n+1}(L)$;

(2) if $\pi \in M E_{n+1}(L)$ and $a_{1}, \ldots, a_{n} \in M E_{0}(L)$, then $\pi\left(a_{1}, \ldots, a_{n}\right) \in M E_{1}(L)$;

(3) if $\phi \in M E_{1}(L)$ and $x_{1}, \ldots, x_{n}$ are distinct individual variables, then $\left[\lambda x_{1} \ldots x_{n} \phi\right] \in M E_{n+1}(L)$;

(4) if $\phi \in M E_{1}(L)$, then $\sim \phi \in M E_{1}(L)$;

(5) if $\phi, \psi \in M E_{1}(L)$, then $(\phi \rightarrow \psi) \in M E_{1}(L)$;

(6) if $\phi \in M E_{1}(L)$ and $a$ is an individual or predicate variable, then $\forall a \phi \in$ $M E_{1}(L)$

(7) if $\phi \in M E_{1}(L)$, then $[\lambda \phi] \in M E_{0}(L)$; and

(8) if $n \in \omega-\{1\}$, then $M E_{n}(L) \subseteq M E_{0}(L)$.

The terms of a language $L$, needless to say, are now all of the members of $M E_{0}(L)$; and for $n>0$, the members of $M E_{n+1}(L)$ are the $n$-place predicate expressions of $L$. Naturally, the wffs of $L$ are now the members of $M E_{1}(L)$. Note that whereas by clause (8) every predicate expression is a term, not every wff is also a term; but then, by clause (3), if $\phi$ is a wff, so is $[\lambda \phi]$, and, by clause (7), $[\lambda \phi]$ is a term. Wffs are terms, in other words, when prefixed by the $\lambda$-operator. (We read $[\lambda \phi]$ as 'that $\phi$ ' when it occurs in a subject position. The subject positions of a wff $\phi$ or of a complex predicate expression containing $\phi$ are of course any one of the subject or argument positions of the predicate expressions occurring in $\phi$.) For convenience, we shall also speak of wffs that are terms as 0-place predicate expressions. We assume the obvious definitions of bondage and freedom of terms in wffs and $\lambda$-abstracts, as well as the notion of one term being free for another in a wff or $\lambda$-abstract.

Now where $\xi$ is a meaningful expression, i.e., $\xi \in M E_{n}(L)$, for some language $L$ and $n \in \omega$, we say that $\xi$ is homogeneously stratified iff there is a function $t$ with the set of terms of $L$ occurring in $\xi$ as domain such that: (1) for all $k \in \omega$, all $k$-place predicate expressions $\pi$ of $L$ and all terms $a_{1}, \ldots, a_{k}$ of $L$, if $\pi\left(a_{1}, \ldots, a_{k}\right)$ is a wff occurring in $\xi$, then (i) $t\left(a_{i}\right)=t\left(a_{j}\right)$, for $1 \leq i \leq$ $j \leq k$, and (ii) $t(\pi)=t\left(a_{1}\right)+1$; and (2) for all $k \in \omega$, all individual variables $x_{1}, \ldots, x_{k}$ and all wffs $\phi$ of $L$, if $\left[\lambda x_{1} \ldots x_{k} \phi\right]$ occurs in $\xi$, then $t\left(x_{i}\right)=t\left(x_{j}\right)$, for $1 \leq i \leq j \leq k$, and $t\left(\left[\lambda x_{1} \ldots x_{k} \phi\right]\right)=t\left(x_{1}\right)+1$. In terms of this notion we say that $\phi$ is a $w f f$ of $\lambda H S T^{*}$ iff $\phi$ is a wff in which every predicate expression of the form $\left[\lambda x_{1} \ldots x_{n} \psi\right]$ is homogeneously stratified. It is clear of course that not every wff is a wff of $\lambda H S T^{*}$.

4 The system $\lambda \mathrm{HST}^{*} \quad$ In turning now to an axiomatization of $\lambda H S T^{*}$ we again take all tautologous wffs (only now of $\lambda H S T^{*}$ as well) and all wffs of $\lambda H S T^{*}$ of the form of $(A 1),(A 2),(A 3)$, and $\left(L L^{*}\right)$ together with all those of the following forms:

$$
\left[\lambda x_{1} \ldots x_{n} P\left(x_{1}, \ldots, x_{n}\right)\right] \equiv P, \text { where } P^{n} \text { is an } n \text {-place predicate }
$$
variable or constant. 
$\left(\lambda\right.$-Conv*) $\quad\left[\lambda x_{1} \ldots x_{n} \phi\right]\left(a_{1}, \ldots, a_{n}\right) \leftrightarrow \phi\left(a_{1} / x_{1}, \ldots, a_{n} / x_{n}\right)$, where each $a_{i}$ is free for $x_{i}$ in $\phi$.

$\left(\operatorname{HSCP}_{\lambda}^{*}\right) \quad \exists F^{n}\left(\left[\lambda x_{1} \ldots x_{n} \phi\right] \equiv F\right)$, where $F^{n}$ does not occur free in $\phi$.

Note that since only wffs of $\lambda H S T^{*}$ are involved here, the $\lambda$-abstracts which occur in instances of $\left(\lambda-C_{\text {Conv }}{ }^{*}\right)$ and $\left(H S C P_{\lambda}^{*}\right)$ must be homogeneously stratified. As inference rules we again take modus ponens and universal generalization (as applied to any variable of any type) and we assume that theoremhood and derivability from premises are defined as in $H S T^{*}$.

By $\left(L L^{*}\right)$ and $(A 3)$, the principle $\left(U I_{1}^{*}\right.$ of universal instantiation of any term for a generalized individual variable is also provable in $\lambda H S T^{*}$. Similarly, by $\left(L L^{*}\right)$ and $\left(H S C P_{\lambda}^{*}\right)$, the principle of universal instantiation of an $n$-place $\lambda$-abstract of $\lambda H S T^{*}$ for a generalized $n$-place predicate variable is also provable in $\lambda H S T^{*}$ :

(UI $\left.\mathbf{I}_{\lambda}^{*}\right) \quad \forall F^{n} \psi \rightarrow \psi\left(\left[\lambda x_{1} \ldots x_{n} \phi\right] / F^{n}\right)$,

and therefore, by $\left(I d_{\lambda}^{*}\right.$ and $\left(L L^{*}\right)$, so is the principle $\left(U I_{n}^{*}\right)$. Of course, also provable in $\lambda H S T^{*}$, by $\left(L L^{*}\right)$, universal generalization, and elementary transformations, is the thesis $\left(\right.$ Ind $\left.^{*}\right)$.

The converse of $\left(\right.$ Ind $\left.^{*}\right)$, viz., the principle of extensionality:

$\left(\mathbf{E x t}_{\mathbf{n}}^{*}\right) \quad \forall F^{n} \forall G^{n}\left(\forall x_{1} \ldots \forall x_{n}\left[F\left(x_{1}, \ldots, x_{n}\right) \leftrightarrow G\left(x_{1}, \ldots, x_{n}\right)\right] \rightarrow F \equiv G\right)$,

is not provable in $\lambda H S T^{*}$, however, which is as it should be except for those theories which explicitly assume otherwise. (Frege would assume $\left(E x t^{*}\right)$, though on his interpretation nominalized predicates do not denote concepts but conceptcorrelates.) We would clearly want to reject $\left(E x t^{*}\right)$, for example, if we were to introduce the modal operator $\square$ into the grammar of $\lambda H S T^{*}$ along with, say, the axioms of $S 5$ modal propositional logic, the Carnap-Barcan wff for quantifiers and $\square$, and the rule of modal generalization into $\lambda H S T^{*}$ itself - calling the result $\square \lambda H S T^{*}$. In that case, however, it is not clear that we would also want to reject the corresponding principle of intensionality ${ }^{2}$ :

$\left(\square \mathbf{E x t}_{\mathbf{n}}^{*}\right) \quad \forall F^{n} \forall G^{n}\left(\square \forall x_{1} \ldots \forall x_{n}\left[F\left(x_{1}, \ldots, x_{n}\right) \leftrightarrow G\left(x_{1}, \ldots, x_{n}\right)\right] \rightarrow F \equiv G\right)$.

One of the benefits of assuming either $\left(E x t^{*}\right)$ or $\left(\square E x t^{*}\right)$ is that we are able on that basis to eliminate all occurrences of the $\lambda$-operator; that is, the wffs of $\lambda H S T^{*}$ are in that case reducible to those of $\left.H S T^{*}\right)$. Thus, using $\left(L L^{*}\right)$, $\left(U I_{2 \lambda}^{*}\right),\left(\lambda-\right.$ Conv $\left.^{*}\right)$ and elementary transformations, the following lemma is easily provable. (We use ' $(\square)$ ' to indicate the corresponding lemma in case $\left(\square E x t^{*}\right)$ and $\square \lambda H S T^{*}$ are used instead of $\left(E x t^{*}\right)$ and $\left.\lambda H S T^{*}.\right)$

Lemma $^{3} \quad$ If $\phi, \psi$ are $w f f s$ of $\lambda H S T^{*}$ and $F^{n}$ does not occur in $\phi$ or $\psi$, then:

$$
\begin{gathered}
\left((\square) E x t^{*}\right) r_{(\square) \lambda H S T^{*}} \phi \leftrightarrow \exists F^{n}\left[(\square) \forall x_{1} \ldots \forall x_{n}\left(F\left(x_{1}, \ldots, x_{n}\right) \leftrightarrow \psi\right)\right. \\
\left.\& \phi\left(F^{n} /\left[\lambda x_{1} \ldots x_{n} \psi\right]\right)\right] .
\end{gathered}
$$

Accordingly, since the $\lambda$-abstracts occurring in any wff $\phi$ of $\lambda H S T^{*}$ always occur in certain subwffs of $\phi$ relative to which those occurrences are free (even 
if they are not free in the broader context of $\phi$ itself), then by systematically interchanging these subwffs with $\lambda$-free subwffs on the basis of the above lemma, we are able to establish the following $\lambda$-elimination theorem. ${ }^{4}$

$\lambda$-Elimination Theorem For every $w f f \phi$ of $\lambda H S T^{*}$ there is a $\lambda$-free $w f f \psi$ of $\lambda H S T^{*}$ such that $\psi$ is homogeneously stratified if $\phi$ is, and

$$
\left((\square) E x t^{*}\right) \frac{1(\square) \lambda H S T^{*}}{(\phi \leftrightarrow \psi) . ~}
$$

It follows, accordingly, that if $\lambda H S T^{*}+\left(E x t^{*}\right)$ is a conservative extension of $H S T^{*}+\left(E x t^{*}\right)$ and $H S T^{*}+\left(E x t^{*}\right)$ is consistent, then so is $(\square) \lambda H S T^{*}+$ $\left((\square) E x t^{*}\right)$. (Note that $\square \lambda H S T^{*}+\left(\square E x t^{*}\right)$ is easily shown to be consistent if $\lambda H S T^{*}+\left(E x t^{*}\right)$ is consistent, by simply interpreting $\square$ as double negation.) We assume in the statement of the following theorem what we show in Section 6; viz., that $\lambda H S T^{*}+\left(E x t^{*}\right)$ is a conservative extension of $H S T^{*}+$ $\left(E x t^{*}\right)$.

Theorem If HST $T^{*}+\left(E x t^{*}\right)$ is consistent, then so is $(\square) \lambda H S T^{*}+\left((\square) E x t^{*}\right)$.

In [2], incidentally, we showed that $H S T^{*}$ is consistent if monadic $H S T^{*}$ is; and, furthermore, by using a result from [4], that monadic $H S T^{*}+\left(E x t^{*}\right)$ is consistent if weak Zermelo set theory is consistent. (Weak Zermelo set theory is the restriction of Zermelo set theory to those instances of the Aussonderungsaxiom in which all quantifiers are limited or restricted, i.e., have the forms $(\forall x \in y) \phi$ or $(\exists x \in y) \phi$.) Accordingly, it follows that if weak Zermelo set theory is consistent, then so is $(\square) \lambda H S T^{*}+\left((\square) E x t^{*}\right)$.

Theorem If weak Zermelo set theory is consistent, then so is ( $\square) \lambda H S T^{*}+$ $\left((\square) E x t^{*}\right)$.

Finally, where $n \neq k$, it might be noted that despite the apparent plausibility of

$\left(\right.$ Inf $\left.^{*}\right) \quad \forall F^{n} \forall G^{k}(F \not \equiv G)$

as a thesis of $H S T^{*}$ or $\lambda H S T^{*}$, it is not a theorem of either. We have avoided taking it as a basic axiom schema here only because the set of its instances amounts to requiring that there be at least a potential infinity of individuals. The thesis is plausible, moreover, primarily only for the Platonist who identifies the individual denoted by a nominalized predicate with the property or relation designated by that predicate when it occurs in a predicate position. It need not be accepted by a Fregean, for example, who denies that concepts are individuals and who interprets nominalized predicates as standing not for concepts but for concept-correlates. In either case, however, it can be shown that if weak Zermelo set theory together with an axiom of infinity is consistent then so is $\lambda H S T^{*}+\left(\operatorname{Inf} f^{*}\right)$.

5 The system $\boldsymbol{H S T}_{\lambda}^{*} \quad$ We turn now to our second goal, viz., the formulation of a system $H S T_{\lambda}^{*}$ which does not restrict itself grammatically to homogeneously stratified $\lambda$-abstracts but which takes only those properties and relations to be individuals that are already posited as such in $H S T^{*}$. The fulfillment of 
this goal, however, at least insofar as every $\lambda$-abstract (homogeneously stratified or not) will express a concept (as a value of the bound predicate variables), requires that we drop the (axiomatic) assumption $(A 3)$ that every nominalized predicate expression actually denotes an individual (as a value of the bound individual variables). That is, the quantificational logic contained in $H S T_{\lambda}^{*}$ must be free of existential presuppositions regarding singular terms. We do this initially by replacing $(A 3)$ with

(A3') $\quad \forall y \exists x(x \equiv y)$,

and allow for the possible truth of $\sim \exists x(a \equiv x)$.

This is not the only change that will be required, however; e.g., $\left(I n d^{*}\right)$ will no longer be validated except in the qualified form:

$\left(\mathbf{3} / \mathbf{I n d}_{\mathbf{n}}^{*}\right) \quad \forall F^{n} \forall G^{n}\left(\exists y(F \equiv y) \&(F \equiv G) \rightarrow \forall x_{1} \ldots \forall x_{n}\left[F\left(x_{1}, \ldots, x_{n}\right) \leftrightarrow\right.\right.$ $\left.\left.G\left(x_{1}, \ldots, x_{n}\right)\right]\right)$,

and therefore $\left(L L^{*}\right)$ will no longer hold in general either, though $\left(L L_{1}^{*}\right)$, i.e., Leibniz' law for subject position occurrences, remains unproblematic. $\left(L L_{1}^{*}\right)$ and $\left(\exists / I n d^{*}\right)$ will of course validate the following qualified form of $\left(L L^{*}\right)$ :

$\left(\mathbf{3} / \mathbf{L L} \mathbf{L}^{*}\right) \quad \exists x(a \equiv x) \rightarrow[a \equiv b \rightarrow(\phi \leftrightarrow \psi)]$,

where $x$ is not free in $a$, and $\psi$ is obtained from $\phi$ by replacing one or more free occurrences of $a$ by free occurrences of $b$.

Note that since every $\lambda$-abstract will express a concept (as a value of the bound predicate variables), the following comprehension principle:

$\left(\mathbf{C P}_{\lambda}^{*}\right) \quad \exists F^{n}\left(\left[\lambda x_{1} \ldots x_{n} \phi\right] \equiv F\right)$

will be valid even in those cases where

$$
\sim \exists y\left(\left[\lambda x_{1} \ldots x_{n} \phi\right] \equiv y\right)
$$

is provable (such as will hold for the $\lambda$-abstract $[\lambda x \exists G(x \equiv G \& \sim G(x))]$ ). However, since $\left(L L^{*}\right)$, and $\left(I n d^{*}\right)$ in particular, fails in general, $\left(C P_{\lambda}^{*}\right)$ will not suffice to validate $\left(U I_{2 \lambda}^{*}\right)$ (as applied now to arbitrary $\lambda$-abstracts). $\left(U I_{2 \lambda}^{*}\right)$ is valid, however, and it trivially implies $\left(C P_{\lambda}^{*}\right)$ as well as

$$
\exists F^{n}\left(\left[\lambda x_{1} \ldots x_{n} \phi\right] \equiv F \& \forall x_{1} \ldots \forall x_{n}\left[F\left(x_{1}, \ldots, x_{n}\right) \leftrightarrow \phi\right]\right),
$$

and for this reason we will take $\left(U I_{2 \lambda}^{*}\right)$ rather than $\left(C P_{\lambda}^{*}\right)$ as an axiom schema of $H S T_{\lambda}^{*}$. Similarly, insofar as $\left(\exists / I n d^{*}\right)$ does not suffice to validate

$\left(\mathbf{g} / \mathbf{I d}_{\lambda}^{*}\right) \quad\left[\lambda x_{1} \ldots x_{n} P\left(x_{1}, \ldots, x_{n}\right)\right] \equiv P \&\left[P\left(a_{1}, \ldots, a_{n}\right) \leftrightarrow\left[\lambda x_{1} \ldots x_{n} P\left(x_{1}, \ldots\right.\right.\right.$, $\left.\left.\left.x_{n}\right)\right]\left(a_{1}, \ldots, a_{n}\right)\right]$,

we shall replace $\left(I d_{\lambda}^{*}\right)$ by $\left(\exists / I d_{\lambda}^{*}\right)$.

Finally, although

$$
\left[\lambda x_{1} \ldots x_{n} \phi\right]\left(a_{1}, \ldots, a_{n}\right) \rightarrow \phi\left(a_{1} / x_{1}, \ldots, a_{n} / x_{n}\right)
$$

remains valid, where each $a_{i}$ is free for $x_{i}$ in $\phi$, nevertheless its converse fails to also be valid. (A $\lambda$-abstract binds individual variables, after all, and therefore can be true only of values of the bound individual variables.) However, where 
no $x_{i}$ is free in any $a_{j}$, $\left(\lambda-C o n v^{*}\right)$ can be validated in the following qualified form:

$\left(\exists / \lambda-\right.$ Conv $\left.^{*}\right)$

$\left[\lambda x_{1} \ldots x_{n} \phi\right]\left(a_{1}, \ldots, a_{n}\right) \leftrightarrow \exists x_{1} \ldots \exists x_{n}\left(a_{1} \equiv x_{1} \& \ldots \& a_{n} \equiv\right.$ $\left.x_{n} \& \phi\right)$.

Because arbitrary wffs with arbitrary $\lambda$-abstracts are now allowed, we need the following notion in order to stipulate that the properties and relations of $H S T^{*}$ are individuals in $H S T_{\lambda}^{*}$. Accordingly, we say that a meaningful expression $\xi$ is bound to individuals iff for all $n \in \omega$, all $n$-place predicate variables $F^{n}$, and all wffs $\psi$, if $\forall F^{n} \psi$ is a subwff of $\xi$, then for some individual variable $x$ and some wff $\chi, \psi$ is $\left[\exists x\left(F^{n} \equiv x\right) \rightarrow \chi\right]$. (Semantically, this amounts to stipulating that the bound predicate variables occurring in $\xi$ refer only to properties and relations which are individuals. Such a notion is pointless in $\lambda H S T^{*}$, needless to say, since by $(A 3)$ every wff of $\lambda H S T^{*}$ is provable in that system to a wff which is bound to individuals.) The relevant axiom, accordingly, is the following nominalized version of $\left(H S C P_{\lambda}^{*}\right)$ :

$\left(\exists /\right.$ HSCP $\left._{\lambda}^{*}\right) \quad \exists y\left(a_{1} \equiv y\right) \& \ldots \& \exists y\left(a_{k} \equiv y\right) \rightarrow \exists y\left(\left[\lambda x_{1} \ldots x_{n} \phi\right] \equiv y\right)$,

where $\left[\lambda x_{1} \ldots x_{n} \phi\right]$ is homogeneously stratified, $\phi$ is bound to individuals, $y$ is an individual variable not occurring free in $\phi$, and $a_{1}, \ldots, a_{k}$ are all the variables or nonlogical constants occurring free in $\left[\lambda x_{1} \ldots x_{n} \phi\right]$.

Finally, by an axiom of $H S T_{\lambda}^{*}$ we understand any wff which is tautologous or of one of the forms $(A 1),(A 2),\left(A 3^{\prime}\right),\left(L L_{1}^{*}\right),\left(\exists / I n d^{*}\right),\left(\exists / I d_{\lambda}^{*}\right),(\exists /$ $\lambda$-Conv $\left.{ }^{*}\right),\left(U I_{2 \lambda}^{*}\right)$, or $\left(\exists / H S C P_{\lambda}^{*}\right)$. (Again, it should be emphasized that we are dealing now with all wffs, whether they are also wffs of $\lambda H S T^{*}$ or not, i.e., whether the $\lambda$-abstracts they contain are homogeneously stratified or not. This is important since when restricted to the wffs of $\lambda H S T^{*}$ every axiom of $H S T_{\lambda}^{*}$ is a theorem of $\lambda H S T^{*}$. The instances of $(A 3)$ which are refutable in $H S T_{\lambda}^{*}$, in other words, all involve $\lambda$-abstracts which are not homogeneously stratified.) We take modus ponens and universal generalization to be the only inference rules of $H S T_{\lambda}^{*}$, and we assume that theoremhood and derivability from premises is defined for $H S T_{\lambda}^{*}$ in the way it is defined for $\lambda H S T^{*}$.

Note that since $(A 3)$ is not an axiom of $H S T_{\lambda}^{*}$, the principle $\left(U I_{1}^{*}\right)$ of universal instantiation of any term for a generalized individual variable is not provable in $H S T_{\lambda}^{*}$ except in the following qualified form:

$\left(\mathbf{\exists} / \mathbf{U I}_{1}^{*}\right)$

$$
\exists y(a \equiv y) \rightarrow[\forall x \phi \rightarrow \phi(a / x)],
$$

where $a$ is free for $x$ in $\phi$ and $y$ is an individual variable which does not occur free in $a$. By $\left(U I_{2 \lambda}^{*}\right)$ and $\left(\exists / I d_{\lambda}^{*}\right)$, however, $\left(U I_{n}^{*}\right)$ is provable in $H S T_{\lambda}^{*}$ without qualification.

The second-order universal instantiation thesis $\left(U I_{2 \lambda}^{*}\right)$, incidentally, should not be confused with the different principle:

(UI $\left.\mathbf{I}_{2}^{*}\right) \quad \forall F^{n} \phi \rightarrow \check{S}_{\psi}^{F\left(x_{1}, \ldots, x_{n}\right)} \phi \mid$,

which is not provable in $H S T_{\lambda}^{*}$ and in fact is refutable for certain instances. E.g., by $\left(\exists / \lambda-\right.$ Conv $\left.^{*}\right)$,

$$
\vdash_{H S T_{\lambda}^{*}}[\lambda x F(x)](a) \rightarrow \exists x(a \equiv x),
$$


where $x$ is not free in $a$; and therefore by $\left(\exists / I d_{\lambda}^{*}\right)$ and $(U G)$,

$$
\vdash_{H S T_{\lambda}^{*}} \forall F[F(a) \rightarrow \exists x(a \equiv x)] .
$$

That is, according to $H S T_{\lambda}^{*}$, only real individuals have properties (which is not the same as to say that wffs containing denotationless singular terms cannot be true). Thus, if per contra the above universal instantiation principle $\left(U I_{2}^{*}\right)$ of wffs for predicate variables were provable in $H S T_{\lambda}^{*}$, then by substituting $\sim \exists x(a \equiv x)$ for $F(a)$ in the above theorem, it would follow that $(A 3)$ is provable in $H S T_{\lambda}^{*}$ after all; i.e., every term would in that case denote a real individual according to $H S T_{\lambda}^{*}$.

With arbitrary $\lambda$-abstracts, however, there are terms for which it is provable that they must fail to denote a real individual. In particular, it is provable in $H S T_{\lambda}^{*}$ that there is no such individual as the Russell property:

$$
\vdash_{H S T_{\lambda}^{*}} \sim \exists y([\lambda x \exists G(x \equiv G \& \sim G(x))] \equiv y),
$$

and similarly that there is no such individual as the Russell (binary) relation:

$$
\vdash_{H S T_{\lambda}^{*}} \sim \exists y([\lambda x z \exists R(x \equiv R \& \sim R(x, z))] \equiv y) ;
$$

and therefore the above instantiation principle $\left(U I_{2}^{*}\right)$ is actually disprovable in $H S T_{\lambda}^{*}$ when $\psi$ is $\sim \exists x(a \equiv x)$, where $a$ is either of these $\lambda$-abstracts, and $\phi$ is the above theorem that only real individuals have properties in $H S T_{\lambda}^{*}$.

One interesting consequence of the theorem that only real individuals can have properties, incidentally, is the further thesis that nonreal "individuals" are indiscernible from one another:

$$
\vdash_{H S T_{\lambda}^{*}} \sim \exists x(a \equiv x) \& \sim \exists x(b \equiv x) \rightarrow a \equiv b,
$$

where $x$ is not free in either $a$ or $b$. Thus, in particular, every property which the Russell property has the Russell relation also has, and conversely; that is,

$$
\vdash_{H S T_{\lambda}^{*}}[\lambda x \exists G(x \equiv G \& \sim G(x))] \equiv[\lambda x z \exists R(x \equiv R \& \sim R(x, z))],
$$

and therefore by $\left(E G_{2 \lambda}^{*}\right)$, i.e., by the contrapositive of $\left(U I_{2 \lambda}^{*}\right)$,

$$
\vdash_{H S T_{\lambda}^{*}} \exists F^{1} \exists G^{2}(F \equiv G) \text {. }
$$

In other words, the thesis $\left(I n f^{*}\right)$ is refutable in $H S T_{\lambda}^{*}$. The qualified version of this thesis, however, i.e., where $n \neq k$,

$$
\forall F^{n} \forall G^{k}[\exists x(F \equiv x) \vee \exists x(G \equiv x) \rightarrow F \equiv \equiv],
$$

is not refutable in $H S T_{\lambda}^{*}$ and in fact can be shown to be consistent in $H S T_{\lambda}^{*}$ if $H S T^{*}+\left(I n f^{*}\right)$ is consistent.

Finally, although $\left(I n d^{*}\right)$ holds in $H S T_{\lambda}^{*}$ only in its qualified form, there is no problem about its converse, $\left(E x t^{*}\right)$. That is, although $\left(E x t^{*}\right)$ is not provable in $H S T_{\lambda}^{*}$ (which is as it should be), nevertheless it can be shown that $H S T_{\lambda}^{*}+\left(E x t^{*}\right)$ is consistent if $H S T^{*}+\left(E x t^{*}\right)$ is. Similarly, where $H S T_{\lambda}^{*}$ stands to $H S T_{\lambda}^{*}$ the way $\square \lambda H S T^{*}$ stands to $\lambda H S T^{*}$, i.e., where $H S T_{\lambda}^{*}$ is $H S T_{\lambda}^{*}$ supplemented with the $S 5$ modal propositional logic, the Carnap-Barcan 
wffs for quantifiers and $\square$, and the rule of modal generalization, then it can be shown that $H S T_{\lambda}^{*}+\left(\square E x t^{*}\right)$ is consistent if $H S T^{*}+\left(E x t^{*}\right)$ is.

Given either $\left(E x t^{*}\right)$ or $\left(\square E x t^{*}\right)$, needless to say, we can prove a $\lambda$ elimination theorem for $H S T_{\lambda}^{*}$ just the way we did for $\lambda H S T^{*}$. However, because $\left(U I_{1}^{*}\right)$ does not hold in $H S T_{\lambda}^{*}$ except in its qualified form, the leading lemma for this result must be stated in the following somewhat more complex form.

Lemma If $\phi, \psi$ are $w f f s, F^{n}$ and $y_{1}, \ldots, y_{n}$ do not occur in either $\phi$ or $\psi$, and $\left[\lambda x_{1} \ldots x_{n} \psi\right]\left(a_{1_{1}}, \ldots, a_{1_{n}}\right), \ldots,\left[\lambda x_{1} \ldots x_{n} \psi\right]\left(a_{k_{1}}, \ldots, a_{k_{n}}\right)$ are all the subwffs of $\phi$ in which $\left[\lambda x_{1} \ldots x_{n} \psi\right]$ occurs free in $\phi$ in a predicate position, then where

$$
\begin{gathered}
\chi_{i}={ }_{d f}(\square)\left(F ( a _ { i _ { 1 } } , \ldots , a _ { i _ { n } } ) \leftrightarrow \exists y _ { 1 } \ldots \exists y _ { n } \left[a_{i_{1}} \equiv y_{1}\right.\right. \\
\left.\left.\& \ldots \& a_{i_{n}} \equiv y_{n} \& \psi\left(y_{1} / x_{1}, \ldots, y_{n} / x_{n}\right)\right]\right), \\
\left((\square) E x t^{*}\right) \underset{H S T_{\lambda(\square)}^{*}}{ } \phi \leftrightarrow \exists F^{n}\left[(\square) \forall x_{1} \ldots \forall x_{n}\left(F\left(x_{1}, \ldots, x_{n}\right) \leftrightarrow \psi\right)\right. \\
\left.\& \chi_{1} \& \ldots \& \chi_{k} \& \phi\left(F^{n} /\left[\lambda x_{1} \ldots x_{n} \psi\right]\right)\right] .
\end{gathered}
$$

$\lambda$-Elimination Theorem For each wff $\phi$ there is a $\lambda$-free wff $\psi$ such that $\psi$ is homogeneously stratified if $\phi$ is, and $\left((\square) E x t^{*}\right) \vdash_{H S T_{\lambda(\square)}^{*}}(\phi \leftrightarrow \psi)$.

\section{An extensional Fregean semantics for nominalized predicates A Fregean} interpretation of nominalized predicates, we have already noted, interprets such occurrences as standing not for the concepts these predicates stand for when occurring in predicate positions but for certain individuals called conceptcorrelates. Now whether or not we agree with Frege that what predicates stand for in predicate positions cannot themselves be individuals, the idea of a correlation between such entities and individuals can nevertheless be easily incorporated into the well-known semantics or model theory of standard second-order logic in such a way as to provide a simple and direct semantics for nominalized predicates as well. Of course, because this sort of semantics is extensional to begin with, the one drawback in proceeding in this way is that we validate $\left(E x t^{*}\right)$. (Later, in intensionalizing this semantics, we validate ( $\left.\square E x t^{*}\right)$ instead.)

Now we do not claim that the extensional (and later intensional) frames and models described below provide the intended interpretation of either $\lambda H S T^{*}$ or $H S T_{\lambda}^{*}$ (unless of course one adopts either an extensional or intensional Fregean view of properties and relations as unsaturated entities). What we do claim, however, is that these frames and models serve as a guide into the structure, if not the specific content, of whatever one might take to be the intended interpretation of either $\lambda H S T^{*}$ or $H S T_{\lambda}^{*}$; and in that regard they provide an important and indispensible service which should not be shunned. In particular, because they contain the general frames and models of standard second-order logic, the Fregean frames and models for $\lambda H S T^{*}$ and $H S T_{\lambda}^{*}$ allow us to exploit and utilize the well-known techniques and results of standard model theory. Thus, because of the completeness of $\lambda H S T^{*}+\left(E x t^{*}\right)$ and $H S T_{\lambda}^{*}+\left(E x t^{*}\right)$ with respect to the appropriate classes of Fregean frames and models, we are able to prove the consistency of $H S T_{\lambda}^{*}+\left(E x t^{*}\right)$ (and therefore of $H S T_{\lambda}^{*}$ alone) relative to the already proved consistency of $H S T^{*}+\left(E x t^{*}\right)$ relative to weak Zermelo 
set theory. Of course, once we are assured of the consistency of $H S T_{\lambda}^{*}$ (to the extent that we are assured of the consistency of weak Zermelo set theory), we can, as it were, kick away the Fregean semantical ladder we have climbed and adopt $H S T_{\lambda}^{*}$ as a semantical framework in its own right from which to view the world (especially from the perspective of natural language). For those who do not believe in the reality of this view of the world, on the other hand, the Fregean semantics we provide below can help them better understand, or at least to model, what they take to be the ravings of those who do.

Accordingly, by an extensional Fregean frame let us understand an indexed triple $\mathfrak{A}=\left\langle D, X_{n}, f\right\rangle_{n \in \omega}$ such that (1) $D$ is a nonempty domain of discourse; (2) for $n \in \omega, X_{n}$ is a nonempty subset of $P\left(D^{n}\right)$; and (3) there is a set $D^{\prime}$ such that $D \subseteq D^{\prime}$ and $f$ is a function with $D^{\prime} \cup \bigcup_{n \in \omega} X_{n}$ as domain such that for all $d \in D^{\prime}, f(d)=d$, and for $n \in \omega, K \in X_{n}, f(K) \in D^{\prime}$. We take the function $f$ in this definition to represent the Fregean correlation of objects with concepts. We add to this correlation the identity function on the superset $D^{\prime}$ only for the convenience of the semantic definitions that are to follow. Note that by definition $D^{\prime}=r n g(f)$; i.e., $D^{\prime}$ consists of all the Fregean correlates of the objects and concepts represented in the frame. It is this set, it should be noted, which we shall take as the range of values of the free individual variables. Of course, $D$, the domain of discourse, is taken as the range of values of the bound individual variables, and in frames of models of $\lambda H S T^{*}$ it will turn out that $D=D^{\prime}=r n g(f)$. Note that a frame is standard, incidentally, if $X_{n}=\rho\left(D^{n}\right)$, for all $n \in \omega .^{5}$

Now by an assignment (of values to variables) in an extensional Fregean frame defined as above we understand a function $A$ with the set of variables (of all types) as domain such that: (1) for all $n \in \omega$ and all $F^{n}, A\left(F^{n}\right) \in X_{n}$, and (2) $A(x) \in r n g(f)$, for all individual variables $x$. In addition, where $L$ is a language, $\mathfrak{A}$ is an extensional Fregean frame, and $g$ is a function with $L$ as domain such that: (1) $g\left(P^{n}\right) \in X_{n}$, for all $n$-place predicate constants in $L$ and (2) $g(c) \in r n g(f)$, for all individual constants in $L$, then we say that $I=$ $\langle g, \mathfrak{R}\rangle$ is an (extensional) model for $L$. Also, for convenience, where $n \in \omega$, we identify the set of possible extensions of type $n$ in $\mathfrak{A}$ as follows:

(1) $\operatorname{Ext}_{0,2}=D$

(2) $E x t_{n+1, \mathfrak{A}}=X_{n}$.

If $L$ is a language and $I=\langle g, \mathfrak{A}\rangle$ is an extensional model for $L$, then we shall say that $I$ is a general model for $L$ if there is a semantic function ext $_{I}$ defined for each assignment $A$ in $\mathfrak{A}$ in such a way that ext $_{I, A}$ is a function with $\bigcup_{n \in \omega} M E_{n}(L)$ as domain such that for $n \in \omega$ and $\xi \in M E_{n+1}(L)$, $\operatorname{ext}_{I, A}(\xi) \in E x t_{n+1, \mathscr{A}}$ and also satisfying the following conditions:

(1) if $\xi$ is a variable, then $\operatorname{ext}_{I, A}(\xi)=A(\xi)$; and if $\xi$ is a descriptive constant in $L$, then $\operatorname{ext}_{I, A}(\xi)=g(\xi)$;

(2) if $\xi$ is $\pi\left(a_{1}, \ldots, a_{k}\right)$, where $\pi \in M E_{k+1}(L)$ and $a_{1}, \ldots, a_{k} \in M E_{0}(L)$, then $\operatorname{ext}_{I, A}(\xi)=1$ iff $\left\langle f\left(\operatorname{ext}_{I, A}\left(a_{1}\right)\right), \ldots, f\left(\operatorname{ext}_{I, A}\left(a_{k}\right)\right)\right\rangle \in \operatorname{ext}_{I, A}(\pi)$;

(3) if $\xi$ is $\left[\lambda x_{1} \ldots x_{k} \phi\right]$, where $\phi \in M E_{1}(L)$, then $\operatorname{ext}_{I, A}(\xi)=\left\{\left\langle d_{1}, \ldots\right.\right.$, $\left.\left.d_{k}\right\rangle \in D^{k}: \operatorname{ext}_{I, A\left(d_{1} / x_{1}, \ldots, d_{k} / x_{k}\right)}(\phi)=1\right\}$ 
(4) if $\xi$ is $\sim \phi$, where $\phi \in M E_{1}(L)$, then $\operatorname{ext}_{I, A}(\xi)=1$ iff $\operatorname{ext}_{I, A}(\phi)=0$;

(5) if $\xi$ is $(\phi \rightarrow \psi)$, where $\phi, \psi \in M E_{1}(L)$, then $\operatorname{ext}_{I, A}(\xi)=1$ iff either $\operatorname{ext}_{I, A}(\phi)=0$ or $\operatorname{ext}_{I, A}(\psi)=1$;

(6) if $\xi$ is $\forall a \phi$, where $\phi \in M E_{1}(L)$ and $a$ is a variable of type $k$, then $\operatorname{ext}_{I, A}(\xi)=1$ iff for all $J \in \operatorname{Ext}_{k, \mathfrak{A}}, \operatorname{ext}_{I, A(J / a)}(\phi)=1$; and

(7) if $\xi$ is $[\lambda \phi]$, where $\phi \in M E_{1}(L)$, then $\operatorname{ext}_{I, A}(\xi)=\operatorname{ext}_{I, A}(\phi)$.

If $\mathfrak{A}$ is a standard Fregean frame, needless to say, then such a semantic function as $\operatorname{ext}_{I}$ is easily seen to exist. But whether $\mathfrak{A}$ is standard or not, given such a function we can define the satisfaction and truth of a wff $\phi$ of $L$ as follows:

(1) $A$ satisfies $\phi$ in $I$ iff $\operatorname{ext}_{I, A}(\phi)=1$; and

(2) $\phi$ is true in $I$ iff every assignment in $\mathfrak{U}$ satisfies $\phi$ in $I$.

The semantic function ext , it should be noted, is defined for all meaningful expressions of $L$ and not just for those which are well-formed in $\lambda H S T^{*}$, i.e., those in which every $\lambda$-abstract is homogeneously stratified. For convenience, let us say that a model $I$ is a general model for $L\left(\lambda H S T^{*}\right)$ if there is a semantic function ext $t_{I}$ defined as above for all assignments $A$ in $\mathfrak{A}$ and all meaningful expressions of $L$ which are well-formed in $\lambda H S T^{*}$ regardless whether ext $_{I, A}$ is also defined for expressions containing $\lambda$-abstracts which are not homogeneously stratified. Note that although every general model for $L$ is a general model for $L\left(\lambda H S T^{*}\right)$, not every general model for $L\left(\lambda H S T^{*}\right)$ need also be a general model for $L$.

Whether $I$ is a general model for $L$ or only for $L\left(\lambda H S T^{*}\right)$, note that every instance of $\left(E x t^{*}\right),(A 1),(A 2),\left(A 3^{\prime}\right),\left(L L_{1}^{*}\right),\left(\exists / \lambda-C o n v^{*}\right),\left(\exists / I d_{\lambda}^{*}\right)$, and therefore $\left(I d_{\lambda}^{*}\right)$ as well, for which ext $t_{I}$ is defined is true in $I$. Every instance of $\left(L L^{*}\right)$ for which ext $t_{I}$ is defined would be true in $I$ if every instance of $\left(\right.$ Ind $\left.^{*}\right)$ were true in $I$; but note that there are some general models in which instances of $\left(I n d^{*}\right)$ are false (such as those for the system $T^{*}\left({ }^{*}\right)$ of [1]). Also if every instance of $\left(I n d^{*}\right)$ were true in $I$, then where $\mathfrak{A}=\left\langle D, X_{n}, f\right\rangle_{n \in \omega}$ is the Fregean frame upon which $I$ is based, it would follow that $f 1 X_{n}$ is one-to-one, for all $n \in \omega$. If every instance of $(A 3)$ for which $\operatorname{ext}_{I}$ is defined is also true in $I$, then every instance of $\left(\lambda-C o n v^{*}\right)$ for which ext $t_{I}$ is defined is also true in $I$, and, moreover, $D=\operatorname{rng}(f)$.

Now we shall say that $I$ is a model of $\lambda H S T^{*}+\left(E x t^{*}\right)$ iff for some language $L, I$ is a general model for $L\left(\lambda H S T^{*}\right)$ in which every instance of (A3), $\left(H S C P_{\lambda}^{*}\right)$ and $\left(\right.$ Ind $\left.^{*}\right)$ which is a wff of $L$ and of $\lambda H S T^{*}$ is true. It follows, accordingly, that if $I$ is a model of $\lambda H S T^{*}+\left(E x t^{*}\right)$ then every theorem of $\lambda H S T^{*}+\left(E x t^{*}\right)$ which is a wff of $L$ is true in $I$.

Similarly, we shall say that $I$ is a model of $H S T_{\lambda}^{*}+\left(E x t^{*}\right)$ iff for some language $L, I$ is a general model for $L$ in which every instance of $\left(U I_{2 \lambda}^{*}\right)$, $\left(\exists / H S C P_{\lambda}^{*}\right)$ and $\left(\exists /\right.$ Ind $\left.^{*}\right)$ which is a wff of $L$ is true. Accordingly, if $I$ is a model of $H S T_{\lambda}^{*}+\left(E x t^{*}\right)$, then every theorem of $H S T_{\lambda}^{*}+\left(E x t^{*}\right)$ which is a wff of $L$ is true in $I$. Note, incidentally, that since $\forall F \exists x(F \equiv x)$ is true in any model of $\lambda H S T^{*}+\left(E x t^{*}\right)$ and false in any model of $H S T_{\lambda}^{*}+\left(E x t^{*}\right)$, then no model of the one system is a model of the other. 
Finally, if $\Gamma \cup\{\phi\}$ is a set of wffs, then we define logical consequence in $\lambda H S T^{*}+\left(E x t^{*}\right)$ and $H S T_{\lambda}^{*}+\left(E x t^{*}\right)$, respectively, as follows:

(1) $\Gamma \overline{\overline{\lambda H S T^{*}+\left(E x t^{*}\right)}} \phi$ iff $\Gamma \cup\{\phi\}$ is a set of wffs of $\lambda H S T^{*}$ and for all languages $L$, all general models $I=\langle g, \mathfrak{A}\rangle$ for $L\left(\lambda H S T^{*}\right)$ and all assignments $A$ in $\mathfrak{A}$, if $I$ is a model of $\lambda H S T^{*}+\left(E x t^{*}\right), \Gamma \cup\{\phi\}$ is a set of wffs of $L$, and $A$ satisfies every member of $\Gamma$ in $I$, then $A$ satisfies $\phi$ in $I$; and

(2) $\Gamma \longdiv { \overline { H S T _ { \lambda } ^ { * } + ( E x t ^ { * } ) } } \phi$ iff for all languages $L$, all general models $I=\langle g, \mathfrak{A}\rangle$ for $L$, and all assignments $A$ in $\mathfrak{A}$, if $I$ is a model of $H S T_{\lambda}^{*}+\left(E x t^{*}\right)$, $\Gamma \cup\{\phi\}$ is a set of wffs of $L$, and $A$ satisfies every member of $\Gamma$ in $I$, then $A$ satisfies $\phi$ in $I$.

Because the proof of the following completeness theorems is similar to that for the completeness theorems of the intensional semantics which is to follow we avoid giving the details of the proof here.

Completeness theorem for $\lambda \mathbf{H S T}^{*}+\left(\mathbf{E x t}^{*}\right) \quad$ If $\Gamma \cup\{\phi\}$ is a set of wffs of $\lambda H S T^{*}$, then:

$$
\Gamma \longdiv { \overline { \lambda H S T ^ { * } + ( E x t ^ { * } ) } } \phi \text { iff } \Gamma \cup\left(E x t^{*}\right) \varlimsup_{\lambda H S T^{*}} \phi \text {. }
$$

Completeness theorem for $\mathbf{H S T}_{\lambda}^{*}+\left(\mathbf{E x t}^{*}\right) \quad$ If $\Gamma \cup\{\phi\}$ is a set of $w f f s$, then:

$$
\Gamma \varlimsup_{\overline{H S T_{\lambda}^{*}+\left(E x t^{*}\right)}} \phi \text { iff } \Gamma \cup\left(E x t^{*}\right) \vdash_{H S T_{\lambda}^{*}} \phi \text {. }
$$

Finally, since a model of $\lambda H S T^{*}+\left(E x t^{*}\right)$ is based on what in [2] we called a homogeneously stratified Fregean frame, then it follows that $I$ is a model of $\lambda H S T^{*}+\left(E x t^{*}\right)$ iff it is also a model of $H S T^{*}+\left(E x t^{*}\right)$ as defined in [2]. Accordingly, by the above completeness theorem for $\lambda H S T^{*}+\left(E x t^{*}\right)$ and the completeness theorem for $H S T^{*}+\left(E x t^{*}\right)$ in [2], it follows that a $\lambda$-free wff is a theorem of $\lambda H S T^{*}+\left(E x t^{*}\right)$ iff it is already a theorem of $H S T^{*}+\left(E x t^{*}\right)$; i.e., $\lambda H S T^{*}+\left(E x t^{*}\right)$ is a conservative extension of $H S T^{*}+\left(E x t^{*}\right)$.

Theorem $\lambda H S T^{*}+\left(E x t^{*}\right)$ is a conservative extension of $H S T^{*}+\left(E x t^{*}\right)$.

7 The relative consistency of $H S T_{\lambda}^{*}+\left(E x t^{*}\right)$ to $\lambda H S T^{*}+\left(E x t^{*}\right) \quad$ A completeness theorem, it might be objected, does not show that a system is consistent, and in particular it does not show that there are any models of $\lambda H S T^{*}+\left(E x t^{*}\right)$ or $H S T_{\lambda}^{*}+\left(E x t^{*}\right)$. However, since we have already shown that $\lambda H S T^{*}+\left(E x t^{*}\right)$ is consistent relative to monadic $H S T^{*}+\left(E x t^{*}\right)$, and that monadic $H S T^{*}+\left(E x t^{*}\right)\left(+\left(I n f^{*}\right)\right)$ is consistent relative to weak Zermelo set theory $(+($ inf $))$, then it follows that there is a model of $\lambda H S T^{*}+\left(E x t^{*}\right)$ $\left(+\left(I n f^{*}\right)\right)$ if weak Zermelo set theory $(+(\operatorname{Inf}))$ is consistent. We now show that any model of $\lambda H S T^{*}+\left(E x t^{*}\right)\left(+\left(I n f^{*}\right)\right)$ can be converted into a model of $H S T_{\lambda}^{*}+\left(E x t^{*}\right)\left(+\left(\exists / I n f^{*}\right)\right)$.

Theorem If $\lambda H S T^{*}+\left(E x t^{*}\right)\left(+\left(\operatorname{Inf} f^{*}\right)\right)$ is consistent, then so is HST ${ }_{\lambda}^{*}+$ $\left(\operatorname{Ext}^{*}\right)\left(+\left(\exists / \operatorname{Inf} f^{*}\right)\right)$. 
Proof: Suppose $\lambda H S T^{*}+\left(E x t^{*}\right)\left(+\left(I n f^{*}\right)\right)$ is consistent and that $L$ is any language. Then, by the completeness theorem there is a model $I=\langle g, \mathfrak{A}\rangle$ of $\lambda H S T^{*}+\left(E x t^{*}\right)$, i.e., a general model $I$ for $L\left(\lambda H S T^{*}\right)$ in which every wff of $L$ which is a theorem of $\lambda H S T^{*}+\left(E x t^{*}\right)$ is true. Where $\mathfrak{A}=\left\langle\nu, X_{n}, f\right\rangle_{n \in \omega}$, let $Y_{n}=\mathcal{P}\left(D^{n}\right)$, for $n \in \omega$, and let $f_{\lambda}$ be that function with $D \cup \bigcup_{n \in \omega} Y_{n}$ as domain such that $f_{\lambda}(d)=f(d)=d$, for all $d \in D$, and for $n \in \omega, K \in Y_{n}, f_{\lambda}(K)=f(K)$ if $K \in X_{n}$, and otherwise $f_{\lambda}(K)=\langle K, D\rangle$; and finally let $\mathfrak{A}_{\lambda}=\left\langle D, Y_{n}, f_{\lambda}\right\rangle_{n \in \omega}$. It is clear of course that $\mathfrak{A}_{\lambda}$ is an extensional Fregean frame and that $I_{\lambda}=\langle g$, $\left.\mathfrak{A}_{\lambda}\right\rangle$ is a general model for $L$ (since $\mathfrak{A}_{\lambda}$ is standard). It remains only to show, accordingly, that every wff of $L$ which is an instance of either $\left(U I_{2 \lambda}^{*}\right),(\exists /$ $\left.H S C P_{\lambda}^{*}\right)$ or $\left(\exists / I n d^{*}\right)$ (or of $\left(\exists / I n f^{*}\right)$ if $\left(I n f^{*}\right)$ is true in $\left.I\right)$ is true in $I_{\lambda}$.

(1) We note first that by construction, for all assignments $A$ in $\mathfrak{A}_{\lambda}$ and all $n \in \omega: A$ satisfies $\exists x(y \equiv x)$ in $I_{\lambda}$ iff $A(y) \in D$; and $A$ satisfies $\exists x\left(F^{n} \equiv x\right)$ in $I_{\lambda}$ iff $A\left(F^{n}\right) \in X_{n}$. (Observe that $D^{1} \in X_{1}$ since ${\grave{\lambda H S T^{*}}} \forall y[\lambda x(x \equiv x)](y)$, and therefore for all $d \in D,\langle d\rangle \in D^{1} \in X_{1}$. Thus, if $A(d / x)$ satisfies $(y \equiv x)$ in $I_{\lambda}$, for some $d \in D$, then $\left\langle f_{\lambda}(A(y))\right\rangle \in D^{1}$; and since $f_{\lambda}(A(y))=A(y)$, by definition, then $A(y) \in D$. Similarly, if $A(d / x)$ satisfies $(F \equiv x)$ in $I_{\lambda}$, then $f_{\lambda}\left(A(d / x)\left(F^{n}\right)\right) \in D$, and since $\left.A(d / x)\left(F^{n}\right)=A\left(F^{n}\right)\right)$, then $f_{\lambda}\left(A\left(F^{n}\right)\right) \in D$. But if $A\left(F^{n}\right) \notin X_{n}$, then $f_{\lambda}\left(A\left(F^{n}\right)\right)=\left\langle A\left(F^{n}\right), D\right\rangle$, which is impossible if $f_{\lambda}\left(A\left(F^{n}\right)\right) \in D$. That is, if $A$ satisfies $\exists x(F \equiv x)$ in $I_{\lambda}$, then $A\left(F^{n}\right) \in X_{n}$. The converse direction in both cases is similar but even more trivial.)

(2) By a simple induction on $M E_{n}(L)$, for $n \in \omega$, we note that for all $\xi \in M E_{n}(L)$, if $\xi$ is bound to individuals, every $\lambda$-abstract occurring in $\xi$ is homogeneously stratified, and $A, B$ are assignments in $\mathfrak{A}$ and $\mathfrak{A}_{\lambda}$, respectively, such that for all variables $a$ free in $\xi, A(a)=B(a)$, then $\operatorname{ext}_{I, A}(\xi)=$ $\operatorname{ext}_{I_{\lambda}, B}(\xi)$. (We consider only the quantifier case where $\xi$ is of the form $\forall F^{n} \phi$, for some wff $\phi$ of $L$. Note that since $\xi$ is bound to individuals, then $\phi$ must be of the form $(\exists x(F \equiv x) \rightarrow \psi)$; and therefore, by (1) above and the inductive hypothesis, $\operatorname{ext}_{I_{\lambda}, B}\left(\forall F^{n} \phi\right)=1$ iff for all $K \in X_{n}$, $\operatorname{ext}_{I, A\left(K / F^{n}\right)}(\psi)=1$; i.e., iff $\operatorname{ext}_{I, A}\left(\forall F^{n} \phi\right)=1$.)

(3) Every wff of $L$ which is an instance of $\left(U I_{2 \lambda}^{*}\right)$ is true in $I_{\lambda}$. (This is an immediate consequence of the fact that each $Y_{n}$ of $\mathfrak{A}_{\lambda}$ is "full", i.e., $Y_{n}=$ $P\left(D^{n}\right)$.)

(4) Every wff of $L$ which is an instance of $\left(\exists / H S C P_{\lambda}^{*}\right)$ is true in $I_{\lambda}$. (To show this, assume $A$ is an assignment in $\mathfrak{A}_{\lambda}$ which satisfies $\exists y\left(a_{i} \equiv y\right)$ in $I_{\lambda}$, for each variable or nonlogical constant $a_{i}$ free in $\left[\lambda x_{1} \ldots x_{n} \phi\right)$, where $\left[\lambda x_{1} \ldots x_{n} \phi\right]$ is homogeneously stratified, $\phi$ is bound to individuals, and $y$ is new to $\phi$. Note that by (1) above, $A\left(a_{i}\right) \in D$ if $a_{i}$ is an individual variable, and $A\left(a_{i}\right) \in X_{k}$ if $a_{i}$ is a $k$-place predicate variable, for all $k \in \omega$. Now let $B$ be any assignment in $\mathfrak{A}$ such that $B\left(a_{i}\right)=A\left(a_{i}\right)$ (and of course, by the preceding note, there must be at least one such assignment). Note that by (A3),

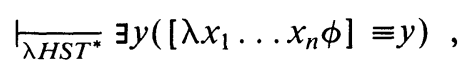

and therefore $B$ satisfies $\exists y\left(\left[\lambda x_{1} \ldots x_{n} \phi\right] \equiv y\right)$ in $I$, from which it follows, by (2) above, that $A$ satisfies $\exists y\left(\left[\lambda x_{1} \ldots x_{n} \phi\right] \equiv y\right)$ in $I_{\lambda}$.) 
(5) Every instance of $\left(\exists / I n d^{*}\right)$ is true in $I_{\lambda}$. This follows from the fact that

$$
\vdash_{H S T_{\lambda}^{*}} \exists y\left(F^{n} \equiv y\right) \&\left(F^{n} \equiv G^{n}\right) \rightarrow \exists y\left(G^{n} \equiv y\right),
$$

and therefore

$$
\begin{gathered}
\vdash_{H S T_{\lambda}^{*}}\left(\exists / \operatorname{Ind}_{n}^{*}\right) \leftrightarrow \forall F^{n}\left[\exists y(F \equiv y) \rightarrow \forall G^{n}(\exists y(G \equiv y) \rightarrow[F \equiv G \rightarrow\right. \\
\left.\left.\left.\forall x_{1} \ldots \forall x_{n}\left[F\left(x_{1}, \ldots, x_{n}\right) \leftrightarrow G\left(x_{1}, \ldots, x_{n}\right)\right]\right]\right)\right] .
\end{gathered}
$$

But the right-hand side of this last theorem is a wff bound to individuals and, by (A3), is equivalent in $\lambda H S T^{*}$ to $\left(I n d^{*}\right)$, which, by hypothesis, is true in $I$. Therefore, by (2) above, $\left(\exists / I n d_{n}^{*}\right)$ is true in $I_{\lambda}$.

(6) Every instance of $\left(\exists / \operatorname{Inf} f^{*}\right)$ is true in $I_{\lambda}$ if every instance of $\left(\operatorname{Inf} f^{*}\right)$ is true in $I$. (Note that where $n \neq k$,

$$
\begin{aligned}
& \vdash_{H S T_{\lambda}^{*}} \exists x\left(F^{n} \equiv x\right) \& \sim \exists x\left(G^{k} \equiv x\right) \rightarrow F^{n} \not G^{k}, \\
& \vdash_{H S T_{\lambda}^{*}} \sim \exists x\left(F^{n} \equiv x\right) \& \exists x\left(G^{k} \equiv x\right) \rightarrow F^{n} \equiv G^{k},
\end{aligned}
$$

and therefore by tautologous transformations,

$$
\begin{gathered}
\vdash_{H S T_{\lambda}^{*}}\left[\exists x\left(F^{n} \equiv x\right) \vee \exists x\left(G^{k} \equiv x\right) \rightarrow F^{n} \not \equiv G^{k}\right] \leftrightarrow \\
{\left[\exists x\left(F^{n} \equiv x\right) \& \exists x\left(G^{k} \equiv x\right) \rightarrow F^{n} \not \equiv G^{k}\right] .}
\end{gathered}
$$

Thus, by universal generalization and distribution:

$$
\vdash_{H S T_{\lambda}^{*}}\left(\exists / \operatorname{Inf} f^{*}\right) \leftrightarrow \forall F^{n} \forall G^{k}\left[\exists x(F \equiv x) \& \exists x(G \equiv y) \rightarrow F^{n} \not \equiv G^{k} .\right.
$$

But the right-hand side here is bound to individuals and, by (A3), is equivalent to $\left(\operatorname{Inf} f^{*}\right)$ in $\lambda H S T^{*}$. That is, the right-hand side is true in $I$ if $\left(I n f^{*}\right)$ is true in $I$; and therefore by (2) above, the right-hand side is true in $I_{\lambda}$ if $\left(\operatorname{Inf} f^{*}\right)$ is true in $I$. But by (3)-(5), $I_{\lambda}$ is a model of $H S T_{\lambda}^{*}+\left(E x t^{*}\right)$, and therefore $\left(\exists / \operatorname{Inf}{ }^{*}\right)$ is true in $I_{\lambda}$ if $\left(\operatorname{Inf} f^{*}\right)$ is true in $I$.)

8 An intensional Fregean semantics for nominalized predicates Let us now add the necessity operator $\square$ to our list of primitive logical constants (and take $\diamond$ as an abbreviation of $\sim \square \sim$ ). Where $L$ is a language, let us add the following clause to the recursive definition of a meaningful expression of $L$ :

(9) if $\phi \in M E_{1}(L)$, then $\square \phi \in M E_{1}(L)$;

and let us understand all our previous definitions about the wffs of $L$, the wffs of $\lambda H S T^{*}$, etc., to now include expressions with $\square$ as well.

We intensionalize the extensional Fregean semantics given in Section 6 as follows. A doubly indexed triple $\left\langle D, X_{n}, f_{i}\right\rangle_{n \in \omega, i \in W}$ is said to be an intensional Fregean frame if: (1) $D$ and $W$ are nonempty sets, (2) $X_{n} \subseteq \odot\left(D^{n}\right)^{W}$, for $n \in \omega$, and (3) there is a set $D^{\prime}$ such that $D \subseteq D^{\prime}$ and for $i \in W, f_{i}$ is a function with $D^{\prime} \cup \bigcup_{n \in \omega} X_{n}$ as domain such that $f_{i}(d)=d$, for all $d \in D^{\prime}$, and for $n \in \omega, K \in X_{n}, f_{i}(K) \in D^{\prime}$. We take $W$ to be the set of possible worlds (or possible contexts of use) of the frame. Properties and relations (or concepts) are 
then represented by functions from possible worlds to extensions of the relevant type. The frame is said to be standard if $X_{n}=P\left(D^{n}\right)^{W}$, for all $n \in \omega$.

As in the extensional frames we take the set $D$ of an intonsional Fregean frame to be the domain of discourse of "real" individuals, i.e., $D$ is the range of values of bound individual variables. Free individual variables, as in the extensional semantics, are allowed to range over the superset $D^{\prime}$ which by definition is identical with $\bigcup_{i \in W} r n g\left(f_{i}\right)$. Note that we allow nominalized predicates to denote different individuals in different possible worlds. This is allowed in order to accommodate those Fregeans who interpret nominalized predicates as denoting the extensions of the concepts otherwise expressed by these predicates in their role as predicates. (A concept's extension in one possible world, needless to say, need not be the same as its extension in a different possible world.) Having made this allowance, however, we shall not in fact pursue such an interpretation of nominalized predicates here-or rather we shall not when dealing with the intensional models of $\square \lambda H S T^{*}+\left(\square E x t^{*}\right)$ and $H S T_{\lambda \square}^{*}+\left(\square E x t^{*}\right)$. For that purpose we say that an intensional Fregean frame as defined above is rigid if for all $i, j \in W, f_{i}=f_{j}$. It is only on the assumption of rigidity, in other words, that we will be able to validate $\forall F^{n} \forall G^{n}[F \equiv G \rightarrow \square(F \equiv G)]$ and reject $\exists F^{n} \exists G^{n}[F \equiv G \& \diamond(F \not \equiv G)]$.

Note that the domain of discourse of an intensional Fregean frame is common to all the possible worlds (or contexts of use) in the frame. We assume in this regard that being-the-value-of-a-bound-individual-variable is a semantic property which corresponds only to the being of an individual and not to its concrete existence, where the latter is a property which an individual can have in some possible worlds and not have in others. Thus if properties and relations are individuals, then their being as individuals should not be identified with their existence in a given possible world; and, indeed, if by existence we mean (world bound) concrete existence, then properties and relations are individuals which cannot exist. (Cf. [3] for a development of such a view.) The individuals denoted by nominalized predicates, in other words, all have being (as values of the bound individual variables), but it might be that none of these individuals ever exist if existence can be a property only of concrete individuals. We shall not concern ourselves here, however, with the representation of (concrete) existence, and we leave its analysis to more specialized applications of $\square \lambda H S T^{*}$ or $H S T_{\lambda \square}^{*}$.

Now where $\mathfrak{A}=\left\langle D, X_{n}, f_{i}\right\rangle_{n \in \omega, i \in W}$ is an intensional Fregean frame, we say that $A$ is an assignment in $\mathfrak{A}$ if $A$ is a function with the set of variables as domain such that: (1) for all $n \in \omega$, all $n$-place predicate variables $F^{n}$, $A\left(F^{n}\right) \in X_{n}$ and (2) for all individual variables $x, A(x) \in \bigcup_{i \in W} r n g\left(f_{i}\right)$. In addition, if $L$ is a language and $g$ is a function with $L$ as domain such that for $n \in \omega$ and $P^{n} \in L, g\left(P^{n}\right) \in X_{n}$, and for each individual constant $c \in L$, $g(c) \in \bigcup_{i \in W} r n g\left(f_{i}\right)$, then we say that $I=\langle g, \mathfrak{N}\rangle$ is an intensional model for $L$. Individual constants are interpreted as "rigid designators", incidentally, since we will be concerned below only with rigid intensional Fregean frames. We now identify the set of possible intensions of type $n$ in $\mathfrak{A}$ as follows:

(1) Int $_{0, \mathfrak{A}}=D$,

(2) Int $_{n+1, \mathfrak{A}}=X_{n}$. 
If $L$ is a language and $I=\langle g, \mathfrak{A}\rangle$ is an intensional model for $L$, then we shall say that $I$ is a general intensional model for $L$ if there is a semantic function int $_{I}$ defined for each assignment $A$ in $\mathfrak{A}$ in such a way that $i n t_{I, A}$ is a function with $\bigcup_{n \in \omega} M E_{n}(L)$ as domain such that for $n \in \omega$ and $\xi \in M E_{n+1}(L)$, $\operatorname{int}_{I, A}(\xi) \in \operatorname{Int}_{n+1, \mathfrak{A}}$ and also satisfying the following conditions for all $i \in W$ :

(1) if $\xi$ is a variable, then $\operatorname{int}_{I, A}(\xi)=A(\xi)$; and if $\xi \in L$, then $\operatorname{int}_{I, A}(\xi)=$ $g(\xi)$

(2) if $\xi$ is $\pi\left(a_{1}, \ldots, a_{k}\right)$, where $\pi \in M E_{k+1}(L)$ and $a_{1}, \ldots, a_{k} \in M E_{0}(L)$, then $\operatorname{int}_{I, A}(\xi)(i)=1$ iff $\left\langle f_{i}\left(\right.\right.$ int $\left._{I, A}\left(a_{1}\right)\right), \ldots, f_{i}\left(\right.$ int $\left.\left._{I, A}\left(a_{k}\right)\right)\right\rangle \in$ int $_{I, A}(\pi)(i)$

(3) if $\xi$ is $\left[\lambda x_{1} \ldots x_{k} \phi\right]$, where $\phi \in M E_{1}(L)$, then $\operatorname{int}_{I, A}(\xi)(i)=\left\{\left\langle d_{1}, \ldots\right.\right.$, $\left.d_{k}\right\rangle \in D^{k}:$ int $\left._{I, A\left(d_{1} / x_{1}, \ldots, d_{k} / x_{k}\right)}(\phi)(i)=1\right\}$

(4) if $\xi$ is $\sim \phi$, where $\phi \in M E_{1}(L)$, then int $t_{I, A}(\xi)(i)=1$ iff int $_{I, A}(\phi)(i)=0$;

(5) if $\xi$ is $\square \phi$, where $\phi \in M E_{1}(L)$, then int $t_{I, A}(\xi)(i)=1$ iff for all $j \in W$, int $_{I, A}(\phi)(j)=1$

(6) if $\xi$ is $(\phi \rightarrow \psi)$, where $\phi, \psi \in M E_{1}(L)$, then int $_{I, A}(\xi)(i)=1$ iff either $\operatorname{int}_{I, A}(\phi)(i)=0$ or $\operatorname{int}_{I, A}(\psi)(i)=1$;

(7) if $\xi$ is $\forall a \phi$, where $\phi \in M E_{1}(L)$ and $a$ is a variable of type $k$, then int $_{I, A}(\xi)(i)=1$ iff for all $J \in$ Int $_{k, \mathfrak{U}}$, int $t_{I, A(J / a)}(\phi)(i)=1$; and

(8) if $\xi$ is $[\lambda \phi]$, where $\phi \in M E_{1}(L)$, then $\operatorname{int}_{I, A}(\xi)=\operatorname{int}_{I, A}(\phi)$.

The existence of the semantic function $i n t_{I}$ is assured, needless to say, if $\mathfrak{A}$ is a standard intensional Fregean frame. But, again, whether $\mathfrak{A}$ is standard or not, given such a function we can define satisfaction, truth, and validity in $I$ of a wff $\phi$ of $L$ as follows, where $A$ is an assignment in $\mathfrak{A}$ and $i \in W$ :

(1) A satisfies $\phi$ in I at $i$ iff $\operatorname{int}_{I, A}(\phi)(i)=1$;

(2) $\phi$ is true in I at $i$ iff every assignment in $\mathfrak{A}$ satisfies $\phi$ in $I$ at $i$; and

(3) $\phi$ is valid in $I$ iff for all $j \in W$, $\phi$ is true in $I$ at $j$.

As in Section 6, we observe that $i n t_{I}$ is defined for all meaningful expressions of $L$ and not just for those that are well-formed in $\square \lambda H S T^{*}$. For the restricted class of meaningful expressions let us say that $I$ is a general intensional model for $L\left(\square \lambda H S T^{*}\right)$ if there is a semantic function int $t_{I}$ defined as above for all assignments $A$ in $\mathfrak{A}$ and all meaningful expressions of $L$ which are well formed in $\square \lambda H S T^{*}$ regardless whether int $I_{I}$ is also defined for meaningful expressions of $L$ containing $\lambda$-abstracts which are not homogeneously stratified. Again, we observe that although every general intensional model for $L$ is a general intensional model for $L\left(\square \lambda H S T^{*}\right)$, the converse does not also hold.

Whether $I$ is a general intensional model for $L$ or only for $L\left(\square \lambda H S T^{*}\right)$, however, note that every instance of $\left(\square E x t^{*}\right),(\mathrm{A} 1),(\mathrm{A} 2),\left(\mathrm{A} 3^{\prime}\right),(\exists / \lambda-$ $\left.\operatorname{Conv}^{*}\right),\left(\exists / I d_{\lambda}^{*}\right)$, and $\left(I d_{\lambda}^{*}\right)$ for which $i n t_{I}$ is defined is valid in $I$. If $I$ is based on a rigid intensional Fregean frame, then every instance of $\left(L L_{1}^{*}\right)$ for which int $t_{I}$ is defined is valid in $I$; and, as in Section 6, if every instance of $\left(I n d^{*}\right)$ is also valid in $I$, then every instance of $\left(L L^{*}\right)$ for which $i n t_{I}$ is defined is valid in $I$. Note also, however, that any instance of any one of the following modal schemas for which int $_{I}$ is defined is also valid in $I$ : 
(M1) $\square \phi \rightarrow \phi$

(M2) $\square(\phi \rightarrow \psi) \rightarrow(\square \phi \rightarrow \square \psi)$

(M3) $\quad \diamond \phi \rightarrow \square \diamond \phi$

(M4) $\forall a \square \phi \leftrightarrow \square \forall a \phi$ (where $a$ is any variable).

Accordingly, where $\square \lambda H S T^{*}$ is the result of adding all wffs of $\lambda H S T^{*}$ which are instances of the above modal theses to the axioms of $\lambda H S T^{*}$ (now understood to apply to wffs with $\square$ as well) along with the rule of modal generalization as a new primitive inference rule, we shall say that a general intensional model for $L\left(\square \lambda H S T^{*}\right)$ is an intensional model of $\square \lambda H S T^{*}+\left(\square E x t^{*}\right)$ iff $I$ is based on a rigid intensional Fregean frame and every wff of $L$ which is a wff of $\square \lambda H S T^{*}$ and an instance of (A3), $\left(H S C P_{\lambda}^{*}\right)$, or $\left(I n d^{*}\right)$ is valid in $I$. It follows, of course, that if $I$ is an intensional model of $\square \lambda H S T^{*}+\left(\square E x t^{*}\right)$, then every theorem of $\square \lambda H S T^{*}+\left(\square E x t^{*}\right)$ which is a wff of $L$ is valid in $I$.

Similarly, where $H S T_{\lambda}^{*}$ is $H S T_{\lambda}^{*}$ supplemented with the same modal theses (but for all wffs, not only just those with homogeneously stratified $\lambda$-abstracts) and the new rule of inference, we say that a general intensional model for a language $L$ is an intensional model of $H S T_{\lambda \square}^{*}+\left(\square E x t^{*}\right)$ iff $I$ is based on a rigid intensional Fregean frame and every wff of $L$ which is an instance of $\left(U I_{2 \lambda}^{*}\right),\left(\exists / H S C P_{\lambda}^{*}\right)$ or $\left(\exists / I n d^{*}\right)$ is valid in $I$. Accordingly, if $I$ is an intensional model of $H S T_{\lambda \square}^{*}+\left(\square E x t^{*}\right)$, then every theorem of $H S T_{\lambda \square}^{*}+$ $\left(\square E x t^{*}\right)$ which is a wff of $L$ is valid in $I$.

Before moving on to the completeness theorems for these modal logics it is perhaps worth noting that whatever metaphysical assumption justifies accepting $\left(\square E x t^{*}\right)$ in $H S T_{\lambda \square}^{*}$ also justifies defining necessity as follows:

$$
\square \phi=_{d f}[\lambda \phi] \equiv[\lambda \forall x(x \equiv x)]
$$

These two wffs are provable equivalent in $H S T_{\lambda \square}^{*}+\left(\square E x t^{*}\right)$, in other words, but not in $H S T_{\lambda \square}^{*}$ alone. Thus while adding $\square$ as a new logical primitive is pointless in $H S T_{\lambda \square}^{*}+\left(\square E x t^{*}\right)$, it is not pointless in $H S T_{\lambda \square}^{*}$ alone.

It is not pointless to introduce $\square$ as a new logical primitive of $\square \lambda H S T^{*}+$ $\left(\square E x t^{*}\right)$, incidentally, since in that context the above definition would not suffice to explain the use of $\square$ with wffs that are not homogeneously stratified. That is, as a wff of $\lambda H S T^{*}$ the above definiens would restrict the application of $\square$ to wffs that are homogeneously stratified (since only these wffs can occur within the $\lambda$-abstracts of $\lambda H S T^{*}$ ); and since there are wffs of $\lambda H S T^{*}$, including tautologous wffs, which are not homogeneously stratified, the above definition would in that case fail to validate the rule of modal generalization when applied to any axiom of $\lambda H S T^{*}$.

We assume that the semantic relations of logical consequence in $\square \lambda H S T^{*}+\left(\square E x t^{*}\right)$ and $H S T_{\lambda \square}^{*}+\left(\square E x t^{*}\right)$, respectively, have been defined as in the extensional semantics of Section 6 except for using satisfaction in $I$ at any given world $i$ of $I$ in the definiens in place of the extensional notion of satisfaction in I simpliciter.

Completeness theorem for $\square \lambda$ HST $^{*}+\left(\square \mathbf{E x t}^{*}\right) \quad$ If $\Gamma$ is a set of wffs of $\square \lambda H S T^{*}$ which is consistent in $\square \lambda H S T^{*}+\left(\square E x t^{*}\right)$, then there are a language 
$L$, a general intensional model $I=\langle g, \mathfrak{A}\rangle$ for $L\left(\square \lambda H S T^{*}\right)$ which is an intensional model of $\square \lambda H S T^{*}+\left(\square\right.$ Ext $\left.{ }^{*}\right)$, a possible world $i$ of $\mathfrak{A}$, and an assignment $A$ in $\mathfrak{2}$ such that $A$ satisfies every member of $\Gamma$ in $I$ at $i$.

Completeness theorem for $\mathbf{H S T}_{\lambda \square}^{*}+\left(\square \mathrm{Ext}^{*}\right) \quad$ If $\Gamma$ is a set of $w f f s$ which is consistent in $H S T_{\lambda}^{*}+\left(\square E x t^{*}\right)$, then there are a language $L$, a general intensional model $I=\langle g, \mathfrak{A}\rangle$ for $L$ which is an intensional model of $H S T_{\lambda}^{*}+$ $\left(\square E x t^{*}\right)$, a possible world $i$ of $\mathfrak{A}$, and an assignment $A$ in $\mathfrak{A}$ such that $A$ satisfies every member of $\Gamma$ in I at $i$.

Proof: We shall give a proof here only for $H S T_{\lambda \square}^{*}+\left(\square E x t^{*}\right)$, since the proof for $\square \lambda H S T^{*}+\left(\square E x t^{*}\right)$ is similar (and somewhat simpler since in that case free logic considerations can be ignored). We shall also assume, but only for convenience of presentation, that $\Gamma$ is a set of wffs of a countable language. Maximal consistency (in $H S T_{\lambda \square}^{*}$ ) is defined as usual, and where $L$ is a language we say that $K$ is an $\omega$-complete set of wffs of $L$ if for all variables $a$ and wffs $\psi$ of $L, \exists a \psi \in K$ only if $\psi(b / a) \in K$ and $\exists a(b \equiv a) \in K$, for some term $b$ of $L$ of the same type as $a$ which is free for $a$ in $\psi$.

We now assume the hypothesis and take $c_{0}, \ldots, c_{n}, \ldots(n \in \omega)$ to be pairwise distinct individual constants new to all the wffs in $\Gamma$. Also, for $n \in \omega$, let $P_{0}^{k}, \ldots, P_{n}^{k}, \ldots(n \in \omega)$ be pairwise distinct $k$-place predicate constants new to $\Gamma$, and let $L$ be the set of all these new descriptive constants together with those which occur in the wffs in $\Gamma$. We assume an ordering $\delta_{1}, \ldots, \delta_{n}, \ldots(n \in \omega)$ of all the wffs of $L$ of the form $\exists a \phi$, where $a$ is any variable. We define the chain $\Gamma_{0}, \ldots, \Gamma_{n}, \ldots(n \in \omega)$ by recursion as follows:

(1) $\Gamma_{0}=\Gamma$;

(2) if $\delta_{n+1}$ is $\exists a \phi$ and $\phi$ is not an identity wff $(\xi \equiv a)$, then

$$
\Gamma_{n+1}=\Gamma_{n} \cup\{\exists a \phi \rightarrow \phi(b / a) \& \exists a(b \equiv a)\},
$$

where $b$ is the first constant in $L$ of the same type as $a$ which is new to $\Gamma_{n} \cup\left\{\delta_{n+1}\right\}$;

(3) if $\delta_{n+1}$ is $\exists x(\xi \equiv x)$, then

$$
\Gamma_{n+1}=\Gamma_{n} \cup\{[\exists x(\xi \equiv x) \rightarrow \xi \equiv b],[\sim \exists x(\xi \equiv x) \rightarrow \xi \equiv b]\},
$$

where $b$ is the first individual constant in $L$ which is new to $\Gamma_{n} U$ $\left\{\delta_{n+1}\right\}$.

We observe that, by hypothesis, $\Gamma_{0}$ is consistent in $H S T_{\lambda \square}^{*}+\left(\square E x t^{*}\right)$. Also, if $\Gamma_{n+1}$ is constructed as in clause (2) then by the usual argument $\Gamma_{n+1}$ is consistent in $H S T_{\lambda \square}^{*}+\left(\square E x t^{*}\right)$ if $\Gamma_{n}$ is. If $\Gamma_{n+1}$ is constructed as in clause (3), then it is not consistent only if $(\xi \not \equiv b)$ is derivable from $\Gamma_{n}$ in $H S T_{\lambda \square}^{*}+$ $\left(\square E x t^{*}\right)$; but since $b$ is new to $\xi$ and to $\Gamma_{n}$ then it can be shown that $(\xi \not \xi)$ is also derivable from $\Gamma_{n}$, which is impossible if $\Gamma_{n}$ is consistent in $H S T_{\lambda}^{*}+$ $\left(\square E x t^{*}\right)$. We conclude, accordingly, that $\bigcup_{n \in \omega} \Gamma_{n}$ is consistent and that there is a maximally consistent set $K$ of wffs of $L$ such that $\Gamma_{n} \subseteq K$, for all $n \in \omega$. Note that by construction $K$ is $\omega$-complete. Also, for each $\xi \in M E_{k}(L)$, for $k \in \omega$, there is a descriptive constant $b$ in $L$ also of type $k$ such that $(\xi \equiv b) \in K$. 
Now let $W$ be the set of $\omega$-complete sets $J$ of wffs of $L$ which are maximally consistent in $H S T_{\lambda}^{*}+\left(\square E x t^{*}\right)$ and such that for all wffs $\psi$ of $L$, if $\square \psi \in K$, then $\square \psi \in J$. Of course, by definition, $K \in W$. We also claim that: (I) for all wffs $\psi$ of $L$ and all $J, J^{\prime} \in W$, if $\square \psi \in J$, then $\square \psi \in J^{\prime}$. For if $\square \psi \notin J^{\prime}$, then $\square \psi \notin K$, in which case $\sim \square \psi \in K$ and therefore by (M3) $\square \sim \square \psi \in K$, from which it follows that $\sim \square \psi \in J$, which is impossible by hypothesis. In addition, we claim that: (II) for all $J \in W$ and all wffs $\psi$ of $L$, if $\psi \in J$ and $\sim \square \psi \in J$, then for some $J^{\prime} \in W, \sim \psi \in J^{\prime}$. To prove (II) we return to the above ordering of wffs of $L$ of the form $\exists a \phi$ and define a new recursion as follows:

(1) $\theta_{0}=\sim \psi$

(2) if $\diamond\left(\theta_{0} \& \ldots \& \theta_{n} \& \delta_{n+1}\right) \notin K$, then $\theta_{n+1}=\theta_{n}$

(3) if $\diamond\left(\theta_{0} \& \ldots \& \theta_{n} \& \delta_{n+1}\right) \in K$ and $\delta_{n+1}=\exists a \phi$, then $\theta_{n+1}=\phi(b / a)$, where $b$ is the first constant in $L$ of the same type as $a$ which is new to $\left(\theta_{0} \& \ldots \& \theta_{n} \& \delta_{n+1}\right)$ and such that $\exists a(b \equiv a) \& \diamond\left[\theta_{0} \& \ldots \& \theta_{n} \&\right.$ $\phi(b / a)] \in K$. (Note: by the modal thesis (M4) and the way $K$ was constructed there must be at least one, and therefore a first, such constant b.)

Now it is easily seen by induction that for all $n \in \omega, \diamond\left(\theta_{0} \& \ldots \& \theta_{n}\right) \in K$, and therefore that $\left\{\theta_{0}, \ldots, \theta_{n}\right\}$ is consistent in $H S T_{\lambda \square}^{*}+\left(\square E x t^{*}\right)$. Thus if $\diamond \theta_{0} \notin K$, then $\sim \diamond \theta_{0} \in K$, i.e., then $\square \psi \in K$, and therefore $\square \psi \in J$, which is impossible by assumption. Similarly, if $\diamond\left(\theta_{0} \& \ldots \& \theta_{n}\right) \in K$, then by definition of $\theta_{n+1}$ it follows that $\diamond\left(\theta_{0} \& \ldots \& \theta_{n+1}\right) \in K$. Accordingly where $M=\left\{\theta_{n}\right.$ : $n \in \omega\} \cup\{\square \chi: \square \chi \in K\}$, note that $M$ is consistent in $H S T_{\lambda}^{*}+\left(\square E x t^{*}\right)$. For by the $S 5$ modal axioms $\left[\diamond \phi_{1} \& \square \phi_{2} \rightarrow \diamond\left(\phi_{1} \& \square \phi_{2}\right)\right]$ is a provable schema, and therefore for all $n, k \in \omega, \diamond\left(\theta_{0} \& \ldots \& \theta_{n} \& \square \chi_{1} \& \ldots \& \square \chi_{k}\right) \in K$, where $\square \chi_{i} \in K$, from which it follows that $M$ is consistent as claimed. Consequently, by Lindenbaum's lemma, there is a set $M^{\prime}$ of wffs $L$ which is maximally consistent in $H S T_{\lambda \square}^{*}+\left(\square E x t^{*}\right)$ and such that $M \subseteq M^{\prime}$. But since $\sim \psi$ is $\theta_{0}$, then $\sim \psi \in M^{\prime}$. Of course by construction $M^{\prime}$ is $\omega$-complete in $L$, and therefore $M^{\prime} \in W$, which concludes our argument for (II).

Now where $a$ is a term of $L$, let $[a]=\{b: b$ is a term of $L$ and ( $a \equiv$ $b) \in K\}$, and let $D^{\prime}=\{[a]: a$ is a term of $L\}$ and $D=\left\{[a] \in D^{\prime}\right.$ : for some individual variable $x$ not free in $a, \exists x(a \equiv x) \in K\}$. We observe that by (A3'), modal generalization and (M4), $\forall y \square \exists x(x \equiv y)$ is provable in $H S T_{\lambda \square}^{*}$, and therefore by $\left(\exists / U I_{1}^{*}\right), \exists x(a \equiv x) \rightarrow \square \exists x(a \equiv x)$ is provable in $H S T_{\lambda}^{*}$. Thus, if $[a] \in D$, then $\exists x(a \equiv x) \in J$, for all $J \in W$.

Where $J \in W$, let $\|_{J}$ be that function with the set of predicate expressions of $L$ as domain such that for all $n \in \omega$ and all $n$-place predicate expressions $\pi$ of $\left.L,|\pi|_{J}=\left\{\left\langle\left[a_{1}\right], \ldots, a_{n}\right]\right\rangle \in D^{n}: \pi\left(a_{1}, \ldots, a_{n}\right) \in J\right\}$. Now for $n \in \omega$ let $X_{n}=\{|\pi|: \pi$ is an $n$-place predicate expression of $L\}$; and for $J \in W$, let $f_{J}$ be that function with $D^{\prime} \cup \bigcup_{n \in \omega} X_{n}$ as domain such that for $[a] \in D^{\prime}, f_{J}([a])=$ [a], and for $n \in \omega$ and all $n$-place predicate expressions $\pi$ of $L, f_{J}(|\pi|)=[\pi]$. Finally, let $\mathfrak{A}=\left\langle D, X_{n}, f_{J}\right\rangle_{n \in \omega, J \in W}$; and where $g$ is that function with $L$ as domain such that $g(c)=[c]$, for each individual constant $c$ in $L$, and $g(P)=$ $|P|$, for each predicate constant $P$ in $L$, let $I=\langle g, \mathfrak{A}\rangle$. 
We observe that by construction $\mathfrak{A}$ is a rigid intensional Fregean frame and that $I$ is an intensional model for $L$. We also claim (III) that $I$ is a general intensional model for $L$.

To show (III) note that if $k \in \omega$ and $\xi$ is a term of $L$ of type $k$, then there is a constant $b$ in $L$ also of type $k$ such that $(\xi \equiv b) \in K$-and therefore $(\xi \equiv b) \in J$, for all $J \in W$ since $\square(\xi \equiv b) \in K$ by $\left(L L^{*}\right)$. For if $k>0$, then the existence of such a constant follows by $\left(C P_{\lambda}^{*}\right)$ and the fact that $K$ is $\omega$-complete in $L$; and if $k=0$, then its existence follows from the definition of $\Gamma_{n+1}$ which is contained in $K$. Accordingly, if $A$ is an assignment in $\mathfrak{A}, \xi \in$ $M E_{n}(L)$, for some $n \in \omega$, and $a_{1}, \ldots, a_{k}$ are all the distinct variables occurring free in $\xi$, then there are constants $b_{1}, \ldots, b_{k}$ in $L$ such that, for $1 \leq i \leq k, b_{i}$ is of the same type as $a_{i}$ and $A\left(a_{i}\right)=\left[b_{i}\right]$ if $a_{i}$ is an individual variable and $A\left(a_{i}\right)=\left|b_{i}\right|$ if $a_{i}$ is a predicate variable. Where $b_{1}, \ldots, b_{k}$ are the first such constants we set:

$$
\xi_{A}=_{d f} \xi\left(b_{1} / a_{1}, \ldots, b_{k} / a_{k}\right) .
$$

On the basis of the above identification we now recursively define the semantic function $v l_{A}$ with the set of meaningful expressions of $L$ as domain and such that for $\xi \in M E_{n+1}(L), v l_{A}(\xi) \in X_{n}$. We will then show that $v a l_{A}(\xi)$ fulfills all of the conditions required for the function $\operatorname{int}_{I, A}(\xi)$ in the definition of a general intensional model for $L$.

(1) if $\xi$ is a variable, then $\operatorname{val}_{A}(\xi)=A(\xi)$; and if $\xi \in L$, then $v a l_{A}(\xi)=g(\xi)$;

(2) if $\xi$ is $\pi\left(a_{1}, \ldots, a_{k}\right)$, then $v a l_{A}(\xi)=\left|\left[\lambda \pi\left(a_{1}, \ldots, a_{k}\right)\right]_{A}\right|$;

(3) if $\xi$ is $\left[\lambda x_{1} \ldots x_{n} \phi\right]$, then $v a l_{A}(\xi)=\left|\xi_{A}\right|$;

(4) if $\xi$ is $\sim \phi$, then $\operatorname{val}_{A}(\xi)=\left|[\lambda \sim \phi]_{A}\right|$;

(5) if $\xi$ is $(\phi \rightarrow \psi)$, then $\operatorname{val}_{A}(\xi)=\left|[\lambda(\phi \rightarrow \psi)]_{A}\right|$;

(6) if $\xi$ is $\square \phi$, then $v a l_{A}(\xi)=\left|[\lambda \square \phi]_{A}\right|$;

(7) if $\xi$ is $\forall a \phi$, then $\operatorname{val}_{A}(\xi)=\left|[\lambda \forall a \phi]_{A}\right|$;

(8) if $\xi$ is $[\lambda \phi]$, then $\operatorname{val}_{A}(\xi)=\left|\xi_{A}\right|$.

By induction over the meaningful expressions of $L$ it can be shown that $v_{a l}$ fulfills all the conditions for int $t_{I, A}$. This is immediate of course in the case where $\xi$ is a variable or constant in $L$. Thus, if $\xi$ is $\pi\left(a_{1}, \ldots, a_{k}\right)$, then by definition of $\|_{J}, v a l_{A}(\xi)(J)=1=\{0]$ iff $\left[\lambda \pi\left(a_{1}, \ldots, a_{k}\right)\right]_{A} \in J$, and therefore iff $\pi\left(a_{1}, \ldots, a_{k}\right) \in J$ (since $(\phi \leftrightarrow[\lambda \phi])$ is a provable schema of $\left.H S T_{\lambda \square}^{*}\right)$. But since $\left[\pi\left(a_{1}, \ldots, a_{k}\right) \rightarrow \exists x\left(a_{i} \equiv x\right)\right]$ is provable in $H S T_{\lambda \square}^{*}$, then $\pi\left(a_{1}, \ldots, a_{k}\right) \in$ $J$ iff $\left\langle\left[a_{1_{A}}\right], \ldots,\left[a_{k_{A}}\right]\right\rangle \in\left|\pi_{A}\right|_{J}$; and also since $f_{J}\left(\operatorname{val}_{A}\left(a_{i}\right)\right)=\left[a_{i_{A}}\right]$, then $\operatorname{val}_{A}\left(\pi\left(a_{1}, \ldots, a_{k}\right)\right)(J)=1$ iff $\left\langle f_{J}\left(\operatorname{val}_{A}\left(a_{1}\right)\right), \ldots, f_{J}\left(\operatorname{val}_{A}\left(a_{k}\right)\right)\right\rangle \in \operatorname{val}_{A}(\pi)(J)$.

If $\xi$ is $\left[\lambda x_{1} \ldots x_{n} \phi\right]$, then by definition $\operatorname{val}_{A}(\xi)(J)=\left\{\left\langle\left[a_{1}\right], \ldots,\left[a_{n}\right]\right\rangle \in\right.$ $\left.D^{n}:\left[\lambda x_{1} \ldots x_{n} \phi\right]_{A}\left(a_{1}, \ldots, a_{n}\right) \in J\right\}$. But by $\left(\exists / \lambda-C_{0 n v}{ }^{*}\right),\left[\lambda x_{1} \ldots x_{n} \phi\right]_{A}\left(a_{1}\right.$, $\left.\ldots, a_{n}\right) \in J$ iff $\left\langle\left[a_{1}\right], \ldots,\left[a_{n}\right]\right\rangle \in D^{n}$ and $\phi_{A\left(\left[a_{1}\right] / x_{1}, \ldots,\left[a_{n}\right] / x_{n}\right)} \in J$, and therefore iff $\left\langle\left[a_{1}\right], \ldots,\left[a_{n}\right]\right\rangle \in D^{n}$ and $[\lambda \phi]_{A\left(\left[a_{1}\right] / x_{1}, \ldots,\left[a_{n}\right] / x_{n}\right)} \in J$. But by definition $\operatorname{val}_{A\left(\left[a_{1}\right] / x_{1}, \ldots,\left[a_{n}\right] / x_{n}\right)}(\phi)=\left|[\lambda \phi]_{A\left(\left[a_{1}\right] / x_{1}, \ldots,\left[a_{n}\right] / x_{n}\right)}\right|$, and therefore $\left\{\left\langle a_{1}\right]\right.$, $\left.\left.\ldots,\left[a_{n}\right]\right\rangle \in D^{n}:\left[\lambda x_{1} \ldots x_{n} \phi\right]_{A}\left(a_{1}, \ldots, a_{n}\right) \in J\right\}=\left\{\left\langle\left[a_{1}\right], \ldots,\left[a_{n}\right]\right\rangle \in D^{n}:\right.$ val $\left._{A\left(\left[a_{1}\right] / x_{1}, \ldots,\left[a_{n}\right] / x_{n}\right)}(\phi)(J)=1\right\}$. 
Now if $\xi$ is $\sim \phi,(\phi \rightarrow \psi), \square \phi$ or $[\lambda \phi]$, then it is easily seen by the inductive hypothesis that $\operatorname{val}_{A}(\xi)$ fulfills the corresponding defining clause for $\operatorname{int}_{I, A}(\xi)$. Suppose finally that $\xi$ is $\forall a \phi$, where $a$ is a variable of type $k$. Then by definition $\operatorname{val}_{A}(\xi)(J)=1$ iff $[\lambda \forall a \phi]_{A} \in J$, and therefore iff $\forall a \phi_{A} \in J$. But since every term of $L$ of type $k$ is represented (in an indiscernibility wff) by a constant of type $k$, then by $\left(\exists / U I_{1}^{*}\right)$ and $\left(U I_{2 \lambda}^{*}\right), \forall a \phi_{A} \in J$ iff for all constants $b$ of type $k$, if $\exists a(b \equiv a) \in J$, then $\phi_{A}(b / a) \in J$. Note, however, that $\exists a(b \equiv a) \in J$ iff $\exists a(b \equiv a) \in K$, and therefore in general, iff $b \in$ Int $_{k, \mathfrak{2}}$. Also, since $\phi_{A}(b / a) \in J$ iff $[\lambda \phi]_{A(g(b) / a)} \in J$, then $\phi_{A}(b / a) \in J$ iff $\operatorname{val}_{A(g(b) / a)}(\phi)$ $(J)=1$. It follows, accordingly, that $v a l_{A}(\forall a \phi)(J)=1$ iff for all $u \in \operatorname{Int}_{k, \mathfrak{Q}}$, $v_{a l} l_{A(u / a)}(\phi)(J)=1$. We conclude then that $v_{a l}(\xi)$ does indeed fulfill all the defining conditions for $\operatorname{int}_{I, A}(\xi)$, from which our claim (III) above follows; i.e., $I$ is a general intensional model for $L$.

Now where $A$ is any assignment in $\mathfrak{A}$, it is clear by the definition of $\operatorname{val}_{A}(\xi)$ that if $\xi$ is a wff of $L$, then for all $J \in W, \operatorname{val}_{A}(\xi)(J)=1$ iff $\xi_{A} \in J$. Accordingly, since every axiom of $H S T_{\lambda}^{*}+\left(\square E x t^{*}\right)$ is a member of every $J \in W$, then $\phi$ is an axiom of $H S T_{\lambda \square}^{*}+\left(\square E x t^{*}\right)$ and a wff of $L$ only if $\operatorname{val}_{A}(\phi)(J)=1$, for all $J \in W$. That is, every axiom of $H S T_{\lambda \square}^{*}+\left(\square E x t^{*}\right)$ is valid in $I$. It follows, accordingly, that $I$ is an intensional model of $H S T_{\lambda}^{*}+$ $\left(\square E x t^{*}\right)$.

Finally, where $A$ is that assignment in $\mathfrak{A}$ such that $A(x)=[x]$, for each individual variable $x$, and $A(F)=|F|$, for each predicate variable $F$, then if $\phi$ is a wff of $L$ and $J \in W, \operatorname{val}_{A}(\phi)(J)=1$ iff $\phi \in J$. Accordingly, since $\Gamma \subseteq K$, then for all $\phi \in \Gamma, v_{A}(\phi)(K)=1$, i.e., $A$ satisfies every member of $\Gamma$ in $I$ at $K$.

Theorem If $\Gamma \cup\{\phi\}$ is a set of wffs of $\square \lambda H S T^{*}$, then

$$
\Gamma \overline{\overline{\left.\square \lambda H S T^{*}+\square E x t^{*}\right)}} \phi \text { iff } \Gamma \cup\left(\square E x t^{*}\right) !_{\square \lambda H S T^{*}} \phi \text {. }
$$

Theorem If $\Gamma \cup\{\phi\}$ is a set of $w f f$, then

$$
\Gamma \longdiv { \overline { H S T _ { \lambda } ^ { * } \square + ( \square E x t ^ { * } ) } } \phi \text { iff } \Gamma \cup\left(\square E x t^{*}\right) ঙ_{H S T_{\lambda \square}^{*} \square} \phi \text {. }
$$

9 Identity versus indiscernibility in $\boldsymbol{H S T}_{\lambda}^{*} \quad$ Before concluding this paper, perhaps it should be noted that we can obtain the full form of Leibniz' law in $H S T_{\lambda}^{*}$ if we are willing to introduce the identity sign into our logical grammar as a purely syncategorematic sign and not as a 2-place predicate constant. As a two-place predicate constant, say $I$, the identity sign, by $\left(\exists / I d_{\lambda}^{*}\right)$, would be a legitimate substituend for the bound predicate variable in the thesis that only real individuals have properties or stand in relations. That is, as a two-place predicate constant,

$$
I(a, b) \rightarrow(\exists x)(a \equiv x) \&(\exists x)(b \equiv x)
$$

would be provable in $H S T_{\lambda}^{*}$; and therefore since the Russell property is identical with itself, i.e., since

$$
I(a, a)
$$


would be a basic thesis of $H S T_{\lambda}^{*}$ with identity, for all terms $a$, it would follow that the Russell property is a real individual after all, i.e.,

$$
(\exists x)(a \equiv x)
$$

would be provable for all terms $a$.

If we introduce the identity sign, $=$, as a purely syncategorematic sign, however, we can avoid this problem and obtain Leibniz' law in its unqualified form. It would follow in particular from $\left(L L_{1}^{*}\right)$ (now formulated in terms of $=$ instead of $\equiv)$ and the identity counterpart of $\left(I n d^{*}\right)$ :

$\left(I d^{*} / E x t^{*}\right) \quad \forall F^{n} \forall G^{n}\left(F=G \rightarrow \forall x_{1} \ldots \forall x_{n}\left[F\left(x_{1}, \ldots, x_{n}\right) \leftrightarrow G\left(x_{1}, \ldots, x_{n}\right)\right]\right)$.

Conversely, we can simply assume $\left(L L^{*}\right)$ (now formulated with $=$ instead of $\equiv$ ) and derive $\left(L L_{1}^{*}\right)$ and $\left(I d^{*} / E x t^{*}\right)$ instead. Note, however, that although real individuals are indiscernible in $H S T_{\lambda}^{*}$ with identity if, and only if, they are identical, i.e.,

$$
\overbrace{\left.H S T_{\lambda}^{*}=\right)} \forall x \forall y(x \equiv y \leftrightarrow x=y),
$$

nevertheless it will be provable in $H S T_{\lambda}^{*}$ with identity that there are concepts for any denotative applications of which identity and indiscernibility must diverge; i.e.,

$$
\vdash_{\left.H S T_{\lambda}^{*}=\right)} \exists F^{n} \exists G^{k}(F \equiv G \& F \neq G) .
$$

Thus, e.g., the Russell property $(n=1)$ and the Russell relation $(k=2)$ are (vacuously) indiscernible but are still not identical. Similarly where $n=k$. E.g., since there is no such individual as the Russell relation,

$$
\vdash_{\left.H S T_{\lambda}^{*}=\right)} \sim \exists y([\lambda x z \exists R(x=R \& \sim R(x, z))]=y),
$$

nor such an individual as the predication relation,

$$
\digamma_{H S T_{\lambda(=)}^{*}} \sim \exists y([\lambda x z \exists F(x=F \& F(z))]=y),
$$

then the one has all and only the same properties as the other (viz., none):

$$
\vdash_{H S T_{\lambda(=)}^{*}}[\lambda x z \exists R(x=R \& \sim R(x, z))] \equiv[\lambda x z \exists F(x=F \& F(z))] .
$$

But, not withstanding their (vacuous) indiscernibility, the Russell relation is not identical with the predication relation:

$$
\vdash_{H S T_{\lambda(=)}^{*}}[\lambda x z \exists R(x=R \& \sim R(x, z))] \neq[\lambda x z \exists F(x=F \& F(z))] .
$$

This is because, not being coextensive, as relational concepts the Russell relation and the predication relation cannot even purport to denote the same individual. That is, by $\left(I d^{*} / E x t^{*}\right)$ it is provable that noncoextensive concepts cannot even purport to denote the same individual. Such a conclusion may be philosophically suspect, however; for whereas the (vacuous) truth of an indiscernibility wff $(a \equiv b)$ is completely unproblematic when neither $a$ nor $b$ denote, one can only wonder as to the real significance of the truth of a nonidentity wff 
$(a \neq b)$ when it is logically necessary that neither $a$ nor $b$ can denote (as singular terms). This problem of course does not arise in $\lambda H S T^{*}$ with identity since in that framework all values of bound predicate variables are also values of bound individual variables and identity is provably equivalent with indiscernibility for all terms whatsoever.

With identity as a new logical constant we must of course add the following clause to the recursive definition of a meaningful expression of a language $L$ :

(10) if $a, b \in M E_{0}(L)$, then $(a=b) \in M E_{1}(L)$.

Also, in the definitions of the extension and intension of a meaningful expression of $L$ relative to an extensional and intensional (free) model or interpretation $I$ for $L$ and assignment $A$, we need to add the following clauses:

(7) if $\xi$ is $(a=b)$, where $a, b \in M E_{0}(L)$, then $\operatorname{ext}_{I, A}(\xi)=1$ iff $f^{+}\left(\operatorname{ext}_{I, A}(a)\right)=f^{+}\left(\operatorname{ext}_{I, A}(b)\right)$;

(8) if $\xi$ is $(a=b)$, where $a, b \in M E_{0}(L)$, then for all $i \in W$, int $_{I, A}(\xi)(i)=1$ iff $f_{i}^{+}\left(\right.$int $\left._{I, A}(a)\right)=f_{i}^{+}\left(\right.$int $\left._{I, A}(b)\right)$.

With regard to an axiomatization of $H S T_{\lambda}^{*}$ with identity, we shall say that an axiom of $H S T_{\lambda(=)}^{*}$ is any wff which is either tautologous or of one of the following forms:

(A1) $\forall a(\phi \rightarrow \psi) \rightarrow(\forall a \phi \rightarrow \forall a \psi)$, where $a$ is any variable

(A2) $\phi \rightarrow \forall a \phi$, where $a$ is any variable not occurring free in $\phi$

(A3') $\forall x \exists y(x=y)$

(A4) $\quad(a=a)$, where $a$ is any term

(LL*) $\quad a=b \rightarrow(\phi \leftrightarrow \psi)$, where $\psi$ is obtained from $\phi$ by replacing one or more free occurrences of a by free occurrences of $b$

$\left(\mathbf{I d}_{\lambda}^{*}\right) \quad\left[\lambda x_{1} \ldots x_{n} P\left(x_{1}, \ldots, x_{n}\right)\right]=P$, where $P$ is an $n$-place predicate variable or constant,

( $\exists / \lambda$-Conv*) $\left[\lambda x_{1} \ldots x_{n} \phi\right]\left(a_{1}, \ldots, a_{n}\right) \leftrightarrow \exists x_{1} \ldots \exists x_{n}\left(a_{1}=x_{1} \& \ldots \& a_{n}=\right.$ $\left.x_{n} \& \phi\right)$, where no $x_{i}$ is free in any $a_{j}$; for all $i, j$ such that $1 \leq i, j \leq n$,

$\left(\mathbf{C} \mathbf{P}_{\lambda}^{*}\right) \quad \exists F^{n}\left(\left[\lambda x_{1} \ldots x_{n} \phi\right]=F\right)$, where $F^{n}$ does not occur free in $\phi$,

$\left(\exists / \mathbf{H S C P}_{\lambda}^{*}\right) \quad \exists y\left(a_{1}=y\right) \& \ldots \& \exists y\left(a_{k}=y \rightarrow \exists y\left(\left[\lambda x_{1} \ldots x_{n} \phi\right]=y\right)\right.$, where $\left[\lambda x_{1} \ldots x_{n} \phi\right]$ is homogeneously stratified, $\phi$ is bound to individuals, $y$ is an individual variable not occurring free in $\phi$, and $a_{1}, \ldots, a_{k}$ are all the variables or nonlogical constants occurring free in $\left[\lambda x_{1} \ldots x_{n} \phi\right]$.

Using modus ponens and universal generalization as the only inference rules, note that by $\left(L L^{*}\right)$,

$\left(\mathbf{I d} / \mathbf{E x t}^{*}\right)$
$\left.\left.\left.\ldots, x_{n}\right)\right]\right)$.$\quad \vdash_{H S T_{\lambda(=)}^{*}} \forall F^{n} \forall G^{n}\left(F=G \rightarrow \forall x_{1} \ldots \forall x_{n}\left[F\left(x_{1}, \ldots, x_{n}\right) \leftrightarrow G\left(x_{1}\right.\right.\right.$,

Also, by $\left(L L^{*}\right)$,

$$
\vdash_{H S T_{\lambda(=)}^{*}}\left[\lambda x_{1} \ldots x_{n} \phi\right]=F \rightarrow\left[\psi \leftrightarrow \psi\left(\left[\lambda x_{1} \ldots x_{n} \phi\right] / F^{n}\right)\right]
$$

and therefore by universal generalization, (A1), and $\left(C P_{\lambda}^{*}\right)$,

(UI $\left.\mathbf{I}_{2 \lambda}^{*}\right) \quad \varlimsup_{H S T_{\lambda(=)}^{*}} \forall F \psi \rightarrow \psi\left(\left[\lambda x_{1} \ldots x_{n} \phi\right] / F^{n}\right)$. 
Accordingly, since by $\left(L L^{*}\right)$

$$
\vdash_{H S T_{\lambda(=)}^{*}} a=b \rightarrow a \equiv b,
$$

then every identity-free wff which is a theorem of $H S T_{\lambda}^{*}$ is a theorem of $H S T_{\lambda(=)}^{*}$. Note also that unlike the refutability of $\left(I n f^{*}\right)$ in $H S T_{\lambda}^{*}$, where $n \neq k$,

$\left(\operatorname{Inf}_{=}^{*}\right) \quad \forall F^{n} \forall G^{k}(F \neq G)$

is not refutable in $H S T_{\lambda(=)}^{*}$.

Where $L$ is a language, let us now say that a general free model $I$ for $L$ is a model of $H S T_{\lambda(=)}^{*}+\left(E x t^{*}\right)$ if every wff of $L$ which is an instance of $\left(C P_{\lambda}^{*}\right)$, $\left(\exists / H S C P_{\lambda}^{*}\right)$ or $\left(I d^{*} / E x t^{*}\right)$ is true in $I$. The extensional notion of logical consequence is then understood to be redefined accordingly.

Note now that the general free model $I_{\lambda}=\left\langle g, \mathfrak{U}_{\lambda}\right\rangle$ which is constructed in the relative consistency proof of Section 7 is not only a model of $H S T_{\lambda}^{*}+$ $\left(E x t^{*}\right)$ but is also a model of $H S T_{\lambda(=)}^{*}+\left(E x t^{*}\right)$. This is because the Fregean correlation function $f_{\lambda}$ constructed in that proof is defined in such a way as to be one-to-one even in the outer domain; i.e., $f_{\lambda}(K)=\langle K, D\rangle$, for all $K \in$ $Y_{n}-X_{n}$, where $D, Y_{n}, X_{n}$ are as constructed in that proof. Using this fact and part (1) of that proof it can then be easily seen that $\left(I d^{*} / E x t^{*}\right)$ is true in $I_{\lambda}$ (and so is $\left(I n f_{=}^{*}\right)$ if $\left(I n f^{*}\right)$ is true in $\left.I\right)$. The rest of the proof is otherwise exactly as in Section 7 except of course for replacing all considerations of wffs with $\equiv$ by the corresponding wffs with $=$ instead.

Theorem If $\lambda H S T^{*}+\left(E x t^{*}\right)\left(+\left(I n f^{*}\right)\right)$ is consistent, then so is $H S T_{\lambda(=)}^{*}+$ $\left(E x t^{*}\right)(+(\operatorname{Inf}=))$.

Finally, by the modal or intensional version of $H S T_{\lambda}^{*}$ with identity, let us understand $H S T_{\lambda(=) \square}^{*}$ to be obtained from $\left(H S T_{\lambda(=)}^{*}\right.$ just the way that $H S T_{\lambda \square}^{*}$ was obtained from $H S T_{\lambda}^{*}$. Then, where $L$ is a language, let us say that a general (intensional) free interpretation $I$ for $L$ is an intensional free model of $H S T_{\lambda(=) \square}^{*}+\left(\square E x t^{*}\right)$ iff $I$ is based on a rigid free Fregean frame and every wff of $L$ which is an instance of $\left(C P_{\lambda}^{*}\right),\left(E / H S C P_{\lambda}^{*}\right)$ or $\left(I d^{*} / E x t^{*}\right)$ is always true in $I$. Again, we understand the intensional notion of logical consequence to be redefined accordingly.

Needless to say the proof of the completeness theorem for $H S T_{\lambda(=)}^{*}+$ $\left(\square E x t^{*}\right)$ proceeds in exactly the same way as the proof for $H S T_{\lambda \square}^{*}+\left(\square E x t^{*}\right)$ except that wherever wffs with $\equiv$ were used in that proof we now are to use the corresponding wff with = instead. In part(3) of that proof we also need to show that $\operatorname{int}_{I, A}(a=b)(J)=1$ iff $(a=b) \in J$; but the proof for this quite straightforward.

Completeness theorem for HST $_{\lambda(=) \square}^{*}+\left(\square \mathbf{E x t}^{*}\right) \quad$ If $\Gamma \cup\{\phi\}$ is a set of $w f f s$, then

$$
\Gamma \varlimsup_{H S T_{\lambda(=) \square+\left(\square E x t^{*}\right)}^{*}} \phi \text { iff } \Gamma \cup\left(\square E x t^{*}\right) \overbrace{H S T_{\lambda(=) \square}^{*}} \phi \text {. }
$$

We conclude by noting that from a purely philosophical standpoint we remain unsure whether to recommend $H S T_{\lambda}^{*}$ with or without identity. Without 
identity, the treatment of denotationless nominalized predicates is unproblematic, and with identity there is the question of the real significance or content of the provable and therefore true nonidentity wffs containing such denotationless terms. On the other hand, with identity, albeit as a purely syncategorematic sign, we do have Leibniz' law in its full unqualified form; and perhaps that alone is enough to recommend $H S T_{\lambda}^{*}$ with identity after all. Of course, there is also always $\lambda H S T^{*}$ where indiscernibility suffices as a full characterization of identity and where whatever is the value of a bound predicate variable is also (on its intended interpretation) a value of the bound individual variables. But then the choice between $\lambda H S T^{*}$ and $H S T_{\lambda}^{*}$ with or without identity will depend on which stand one takes in the realism/conceptualism debate (regarding the nature of predication) whereas the choice between $H S T_{\lambda}^{*}$ with and $H S T_{\lambda}^{*}$ without identity seems entirely to be an "in-house" problem for conceptualism on how to interpret true nonidentity wffs with denotationless nominalized predicates. Space precludes our saying more on any of these issues here.

\section{NOTES}

1. Note that where $n \neq k,\left(L L^{*}\right)$ does not validate $F^{n} \equiv G^{k} \rightarrow\left[F^{n}\left(a_{1}, \ldots, a_{n}\right) \leftrightarrow\right.$ $\left.G^{k}\left(a_{1}, \ldots, a_{n}\right)\right]$, since $G^{k}\left(a_{1}, \ldots, a_{n}\right)$ is not well formed, i.e., it is not a wff. Thus, where $\left(L L^{*}\right)$ involves nominalized predicates of different degrees (number of argument positions), then either $a$ occurs free in $\phi$ only in subject positions (and $\psi$ is $\phi$ with these free subject position occurrences of $a$ replaced by free subject position occurrences of $b)$ or $\left(L L^{*}\right)$ reduces to the tautologous wff: $a \equiv b \rightarrow(\phi \leftrightarrow \phi)$.

2. In stating theses in this manner, we always assume that $x_{1}, \ldots, x_{n}$ are pairwise distinct individual variables. Also, by $\left(E x t^{*}\right)$ and $\left(\square E x t^{*}\right)$ we mean the sets of wffs $\left(E x t_{n}^{*}\right)$ and $\left(\square E x t_{n}^{*}\right)$, respectively, for all $n \in \omega$.

3. Although we are only interested in those applications of this lemma in which $\left[\lambda x_{1} \ldots x_{n} \psi\right]$ occurs free in $\phi$, we note that this is not essential to the proof of the lemma since $\phi\left(F^{n} /\left[\lambda x_{1} \ldots x_{n} \psi\right]\right)$ is otherwise just $\phi$ itself. Note that the right-hand side of this provable wff is homogeneously stratified if $\phi$ is.

4. The interchange law for $\lambda H S T^{*}$ is easily proved by an inductive argument for all wffs of $\lambda H S T^{*}$-so long as the wffs interchanged do not occur within the scope of the $\lambda$-operator; and if they do so occur, then the interchange law holds only on the condition that the wffs interchanged are homogeneously stratified. Note that in proving the $\lambda$-elimination theorem, the subwffs of $\phi$ which both contain and occur within $\lambda$-abstracts must be homogeneously stratified (since $\phi$ otherwise would not be a wff of $\lambda H S T^{*}$ ); and therefore such subwffs can be interchanged with any provably equivalent homogeneously stratified wff. The interchange to be effected on the basis of the above lemma, needless to say, concerns only homogeneously stratified wffs if the subwff in question is itself homogeneously stratified.

5. We assume throughout that each natural number is the set of natural numbers less than it, and therefore take 0 to be the empty set and $1=\{0\}$. We take $P\left(D^{n}\right)$ to be the power-set of $D^{n}$, the set of $n$-tuples drawn from $D$; and therefore $P\left(D^{0}\right)=\{0,1\}$. 


\section{REFERENCES}

[1] Cocchiarella, Nino B., "Fregean semantics for a realist ontology," Notre Dame Journal of Formal Logic, vol. 15 (1974), pp. 552-568.

[2] Cocchiarella, Nino B., "The theory of homogeneous simple types as a second order logic," Notre Dame Journal of Formal Logic, vol. 20 (1979), pp. 505-524.

[3] Cocchiarella, Nino B., "Meinong reconstructed versus early Russell reconstructed," Journal of Philosophical Logic, vol. 11 (1982), pp. 183-214.

[4] Jensen, R., "On the consistency of a slight(?) modification of Quine's New Foundations," Synthese, vol. 19 (1968), pp. 250-263.

[5] Quine, W. V. O., "New foundations for mathematical logic," pp. 80-101 in From a Logical Point of View, Harvard University Press, Cambridge, Massachusetts, 1953.

[6] Quine, W. V. O., Mathematical Logic, Harvard University Press, Cambridge, 1958.

[7] Wang, Hao, “A formal system of logic," The Journal of Symbolic Logic, vol. 15 (1950), pp. 25-32.

\section{Department of Philosophy}

Indiana University

Bloomington, Indiana 47405 